\title{
Categorización de conflictos sociales en el ámbito de los recursos naturales: un estudio de las actividades extractivas mediante la minería de textos
}

\author{
Ramiro Albrieu y Gabriel Palazzo
}

\section{Resumen}

Mediante la aplicación de técnicas de minería de textos, se desarrolló una metodología para medir el número de conflictos sociales relacionados con la explotación de recursos naturales no renovables. Este estudio se centra en los conflictos de cuatro países mineros (Australia, Canadá, Chile y Perú) entre 2003 y 2016, sobre la base de más de 20.000 artículos de los principales periódicos de cada país. Se halló una correlación estadísticamente significativa entre el principal índice y las rentas procedentes de la minería como porcentaje del producto interno bruto (PIB). No obstante, estos resultados deben interpretarse con cautela, dado que no se han abordado los problemas de endogeneidad, y los índices podrían presentar sesgos a causa de diversos factores específicos de cada país. El principal resultado del estudio es una base datos con diferentes índices de conflictos menores relacionados con la explotación de recursos naturales no renovables y su cobertura mediática en los cuatro países.

\section{Palabras clave}

Conflicto social, recursos naturales, recursos no renovables, minería, datos estadísticos, Australia, Canadá, Chile, Perú

Clasificación JEL

J23, J24, 033

\section{Autores}

Ramiro Albrieu es Investigador Asociado en el Departamento de Economía del Centro de Estudios de Estado y Sociedad (CEDES). Correo electrónico: ralbrieu@cedes.org.

Gabriel Palazzo es Asistente de Investigación en el Departamento de Economía del Centro de Estudios de Estado y Sociedad (CEDES). Correo electrónico: gabrielmpalazzo@gmail.com. 


\section{Introducción}

La explotación de recursos naturales subterráneos es un tema controvertido. Por un lado, puede incrementar los ingresos públicos y brindar a la economía los recursos necesarios para crecer; es decir, lo que Hirschman (1977) denominaba encadenamientos indirectos. Por otro lado, existe la percepción, tanto en los trabajos sobre el tema como entre el público en general, de que los costos sociales de estas actividades de explotación no se toman lo suficientemente en cuenta cuando los gobiernos (o las empresas) deciden agotar un determinado recurso natural no renovable. Esta preocupación resulta especialmente pertinente en lo que respecta a los efectos de las acciones actuales sobre el bienestar de las futuras generaciones, pero también a los efectos locales contemporáneos.

El objetivo de la agenda sobre el cambio climático y, más específicamente, de los estudios sobre la contabilidad ecológica y sus aplicaciones principales - como el Sistema de Contabilidad Ambiental y Económica (SCAE) desarrollado por las Naciones Unidas-, es resolver el problema de la equidad intergeneracional (Naciones Unidas, s/f). Las estimaciones del Banco Mundial (2009 y 2011) del ahorro neto ajustado abordan el problema intergeneracional corrigiendo la contabilidad nacional estándar del ahorro (la suma del bienestar presente y futuro) y midiendo la inversión en capital humano y físico, el agotamiento de recursos naturales y el daño ambiental provocado por el dióxido de carbono y otras emisiones. Sin embargo, el problema de la equidad intrageneracional ha resultado más difícil de resolver.

En este artículo, se contribuye a la bibliografía sobre explotación y agotamiento de los recursos naturales desarrollando medidas indirectas de los conflictos sociales a nivel nacional y local (o regional). El objetivo principal es llamar la atención sobre los conflictos relacionados con la explotación de recursos naturales a los que deben hacer frente tanto las instituciones públicas como el sector privado. Para ello, se localizaron patrones de palabras relacionados con los conflictos sociales en artículos sobre el sector minero publicados en los principales periódicos de cuatro países que son grandes productores de minerales: Australia, Canadá, Chile y Perú. El aporte fundamental de este trabajo es la creación de una base de datos destinada a la exploración de efectos causales y correlaciones, a fin de garantizar una mejor gestión de dichos conflictos en el futuro.

La ventaja de estos índices es que son capaces de capturar conflictos menores y medir la intensidad de los diferentes conflictos. No obstante, uno de sus puntos débiles es que podrían contener datos sesgados, debido a los diferentes grados de presión ejercida por las industrias extractivas, los gobiernos y otros agentes sobre los medios de comunicación. Sin embargo, si el nivel de cabildeo se mantuviera constante a lo largo del tiempo, los índices de esta muestra de países solo estarían sesgados a nivel nacional, lo que podría resolverse fácilmente mediante el uso de modelos de regresión de efectos fijos. Los problemas surgirían si el nivel de cabildeo variara con el tiempo o estuviera sujeto a choques temporales. No obstante, incluso si existiera algún sesgo, resulta interesante evaluar la cobertura mediática que se ha brindado a los conflictos sociales relacionados con los recursos naturales.

El artículo se estructura de la siguiente manera: la sección II incluye una breve reseña de la bibliografía sobre minería de textos en el ámbito económico y una explicación de la metodología aplicada para medir el conflicto social. En la sección III se presentan los resultados principales a nivel de país, se analizan las disparidades regionales dentro los países y se definen los diferentes niveles de violencia registrados en los índices de conflicto medidos. La sección IV contiene los modelos de regresión utilizados para evaluar la relación entre los conflictos y los ingresos de los países procedentes de la minería, así como su desempeño económico general. Por último, en la sección $\vee$ se presentan las conclusiones. 


\section{Cuantificación de los conflictos sociales en las industrias extractivas}

\section{Revisión bibliográfica}

La metodología utilizada en este artículo se basa en las técnicas de minería de textos. Estas técnicas promueven la investigación computacional y permiten llegar a conclusiones detectando patrones estadísticos mediante el estudio de las palabras presentes en un texto.

Los periódicos digitales contienen enormes cantidades de información cualitativa que puede procesarse mediante programas informáticos para obtener evaluaciones cuantitativas de variables económicas difíciles de cuantificar. Gupta y Lehal (2009, pág. 60) describen las técnicas de minería de textos como el descubrimiento, a través de la computación, de información nueva, anteriormente desconocida, mediante la extracción automática de datos a partir de diferentes fuentes escritas.

La cantidad de trabajos del ámbito de la economía en los que se utilizan estas técnicas ha ido aumentando con el tiempo. Tetlock (2007), uno de los pioneros en esta área, elaboró un índice de pesimismo en los medios, analizando la columna diaria "Abreast of the Market", del Wall Street Journal. Utilizando modelos de vectores autorregresivos (VAR) básicos, descubrió que los valores altos de pesimismo en los medios predicen de manera robusta la presión a la baja en los precios de mercado, seguida por una reversión a los valores fundamentales. Los resultados de Tetlock parecen indicar que las medidas de contenido en los medios pueden servir como indicador indirecto del sentir los inversionistas. En la misma línea, García (2013) elaboró un índice del sentimiento del mercado contando el número de palabras positivas y negativas de dos columnas financieras ("Financial Markets" y "Topics in Wall Street") del New York Times, publicadas diariamente desde 1905 hasta 2005. Como Tetlock (2007), García observó que el contenido publicado en los medios puede predecir el volumen negociado. Por medio de un modelo parsimonioso de series temporales, García también llegó a la conclusión de que el contenido de las noticias ayuda a predecir el rendimiento diario de las acciones, especialmente durante las recesiones. Aromí (2013) utilizó una metodología similar para evaluar la manera en que los flujos de información de los periódicos influyeron en el desempeño del mercado financiero en la Argentina de octubre de 1996 a diciembre de 2012. Aplicó modelos de regresión con series temporales al rendimiento de las acciones, cuyos determinantes son las medidas del sentimiento de los medios elaboradas por él mismo, los retardos de estas variables y los retardos del rendimiento de las acciones, entre otras variables de control. Los resultados de Aromí resultan compatibles con la presencia de participantes en el mercado que reaccionan de manera desproporcionada a los flujos de información. A fin de obtener un índice cuantitativo, en todos estos estudios se utilizó un enfoque basado en el diccionario, contando palabras positivas y negativas para generar un índice numérico.

Baker, Bloom y Davis (2013) se valen de las técnicas de la minería de textos para elaborar un índice que cuantifique la incertidumbre generada en torno a la política económica y sus efectos sobre la demanda de inversión en el contexto de la recesión económica del período 2007-2009 en los Estados Unidos. Mediante una búsqueda automatizada de términos relacionados con la incertidumbre en materia de política económica en textos de diez de los principales periódicos publicados en los Estados Unidos, crearon un índice de incertidumbre de la política económica, a fin de capturar la incertidumbre imperante con respecto a las políticas fiscal, regulatoria, de gasto y monetaria.

Centrándonos en el tema que nos ocupa en este trabajo, los conflictos sociales relacionados con los recursos naturales cuentan con una historia mucho más extensa que la metodología de minería de textos utilizada en este artículo. Desde la década de 1980, se ha venido cuestionando la opinión convencional de que la riqueza de recursos naturales promueve el desarrollo económico. Muchos 
argumentan que esa misma riqueza es la base del subdesarrollo. Sachs y Warner (1995) atribuyen a la "maldición de los recursos naturales" el menor crecimiento de los países que cuentan con una elevada participación en las exportaciones de productos básicos. Más recientemente, Brunnschweiler y Bulte (2009) afirman que esta maldición de los recursos naturales presenta como mínimo tres dimensiones diferentes: i) crecimiento económico más lento, ii) conflictos civiles violentos y iii) regímenes no democráticos.

Diversas razones pueden explicar el vínculo entre los recursos naturales y el malestar social y los conflictos políticos. Algunas hipótesis planteadas por Sachs y Warner (1995) y Leite y Weidmann (1999) argumentan que los países ricos en recursos naturales presentan mayores niveles de desigualdad y polarización social, debido a que la élite dominante, aprovechándose de su poder político, ejerce presión para conseguir recursos, fomentando comportamientos orientados a la captación de rentas. Collier y Hoeffler (1998, 2000 y 2005) analizaron las causas de diferentes guerras civiles y rebeliones y encontraron que la abundancia de recursos naturales parece influir de manera considerable. En Collier y Hoeffler (2004), se contrasta la hipótesis de que las rebeliones pueden explicarse por injusticias graves con la idea de que se originan por la existencia de oportunidades atípicas para crear una organización rebelde. Sus resultados respaldan la hipótesis de la oportunidad. Defienden que la relación positiva entre las rebeliones y la exportación de productos básicos indica que las oportunidades para la depredación (el control de esas exportaciones) generan conflictos y brindan la oportunidad de financiarlos.

De manera similar, Nafziger y Auvinen (2002) y Sinnott, Nash y De la Torre (2010) sugieren que los conflictos pueden originarse cuando los diferentes grupos sociales no reciben lo que consideran justo, lo que es indicativo de la existencia de un Estado depredador, una regulación deficiente y élites que aprovechan los recursos para obtener rentas en lugar de promover el desarrollo económico. Sinnott, Nash y De la Torre (2010) determinan las consecuencias sociales de la explotación minera y petrolera, cuyo potencial para generar tensiones sociales y conflictos es elevado; en muchos casos, debido a sus efectos adversos sobre el medio ambiente y a las malas condiciones laborales. A su vez, la teoría de la enfermedad holandesa y la hipótesis de Prebisch-Singer ofrecen explicaciones subyacentes sobre la razón por la cual la explotación de los recursos naturales y su efecto sobre la economía pueden generar malestar social entre determinados segmentos de la población (Singer, 1950; Prebisch, 1949).

Brunnschweiler y Bulte (2009) sostienen que es necesario instrumentar las variables utilizadas. Los autores encuentran que existe endogeneidad entre ellas y causalidad inversa: la paz reduce la dependencia de un país de los recursos naturales y no es la dependencia de las exportaciones de productos básicos lo que crea conflictos sociales, lo que contradice la tesis de la maldición de los recursos. De hecho, hallan que la abundancia de recursos puede tener un efecto positivo en el crecimiento económico. Mehlum, Moene y Torvik (2006), como Brunnschweiler y Bulte (2009), Arezki y van der Ploeg (2007), Haber y Menaldo (2011) y Leite y Wiedmann (1999), afirman que es la calidad de las instituciones lo que determina si la abundancia de recursos naturales constituye una bendición o una maldición. Por otra parte, de acuerdo con Giordano, Giordano y Wolf (2005) y Evans (2010), la escasez de recursos puede incrementar el riesgo de futuros conflictos sociopolíticos. Robinson, Torvik y Verdier (2006) elaboran un modelo de economía política para analizar el impacto de los recursos naturales en el desarrollo, en el que los políticos permitirían la sobreexplotación de los recursos naturales y después redistribuirían de manera ineficiente los ingresos para tratar de influir en los resultados de las elecciones. No obstante, concluyen que, en los países en los que existen instituciones que limitan la capacidad de los políticos de valerse del clientelismo para influir en las elecciones, los auges de recursos tienden a incrementar los ingresos nacionales.

Hasta el momento de la elaboración de este artículo, pocos trabajos han utilizado la minería de textos para examinar la relación entre la explotación de recursos naturales y los conflictos sociopolíticos. A nuestro entender, la metodología adoptada en PNUD/Fundación UNIR (2012) es la que más se acerca a la nuestra, dado que, para el análisis de los conflictos, también utilizan su cobertura en los medios. Para su estudio de los conflictos sociales en América Latina, recolectaron datos de 54 periódicos 
publicados en 17 países latinoamericanos de octubre de 2009 a septiembre de 2010. Los autores detectaron tres esferas de conflicto diferenciadas: i) la reproducción social (que representa la mayor proporción de conflictos en el período analizado e incluye los relacionados con el trabajo y los salarios, la tierra y los ingresos), ii) los conflictos institucionales (relacionados con las exigencias de implementar mejoras prácticas en la provisión de bienes públicos, la gestión administrativa y la legitimidad de las autoridades públicas) y iii) los conflictos culturales (relacionados con cuestiones ideológicas y políticas, la seguridad pública y el medio ambiente, entre otras cosas).

Otro ejemplo de este tipo es el trabajo de Dube y Vargas (2013), en el que se utilizan artículos de prensa para caracterizar los conflictos civiles violentos en Colombia. Los autores ofrecen pruebas de que el aumento de los precios del petróleo intensificó la violencia en las áreas que transportaban y producían más petróleo, mientras que el descenso del precio del café incrementó la violencia en las municipalidades en las que se cultivaba más café. La diferencia fundamental entre estas dos actividades es la brecha referida a la intensidad del uso de mano de obra. Por un lado, la reducción del precio del café disminuyó los salarios de los trabajadores y redujo el costo del reclutamiento de dichos trabajadores por parte de grupos armados. Esto se denomina efecto de costo de oportunidad. Por otro lado, la crisis del petróleo incrementa sustancialmente los ingresos del gobierno local, lo que anima a los grupos paramilitares a trasladarse a las zonas petrolíferas para controlar estos recursos. Esto es lo que se conoce como efecto de rapacidad. A diferencia de lo que ocurre en el presente estudio, lo que hacen Dube y Vargas es - en lugar de construir una nueva base de datos mediante la técnica de la minería de textos - utilizar un conjunto de datos del Centro de Recursos para el Análisis de Conflictos (CERAC) extraídos de artículos de prensa.

Por último, la estrategia empírica utilizada en este artículo está inspirada en la de Palazzo (2017), quien elaboró índices de conflictos sociales relacionados con la explotación de un amplio conjunto de recursos naturales en la Argentina durante el período 1996-2014. Este trabajo contribuyó enormemente al desarrollo de la metodología y al procedimiento para verificar si dichos índices mostraban de manera fiable algunos hechos estilizados sobre los conflictos civiles relacionados con las actividades agrícolas, mineras, petroleras, pesqueras y forestales en la Argentina.

\section{Estrategia empírica}

Desarrollamos una metodología que mide la cantidad de conflictos sociopolíticos relacionados con la explotación de recursos minerales, y es susceptible de aplicarse a otros recursos naturales. Para ello, se utilizó un modelo de minería de textos de bolsa de palabras, que consistió en contar el número de palabras hostiles presente en cada artículo que hacía referencia a las industrias extractivas en una ubicación y un período de tiempo determinados. El número de artículos sobre conflictos sirve como indicador indirecto del nivel de conflicto en un momento y un lugar determinados. Esta información se utiliza para crear una serie de índices (por ejemplo, el cociente entre las palabras hostiles y el número total de palabras del artículo) para captar la intensidad del conflicto.

La mayor parte de la bibliografía sobre conflictos sociales examina las guerras civiles en las que el número de muertos sirve como medida de la intensidad. Nuestro índice difiere de aquellos elaborados por otros autores, pues ofrece una medida de conflictos menores que tiene en cuenta huelgas, cierres patronales, marchas de protesta y disputas políticas. Los conflictos están relacionados de manera inequívoca con las industrias extractivas, dada la naturaleza de la metodología utilizada.

Los datos se tomaron de Factiva (Dow Jones, 2020), un sitio web en el que se recoge y almacena un gran número de periódicos de todo el mundo y se clasifican los artículos por industria y sector. Elegimos las áreas de explotación de minas y canteras e industrias de metales primarios, para asegurarnos de que todos los artículos estuvieran relacionados con el sector que pretendemos analizar. 
Idealmente, debería haberse consultado más de un periódico por país, para evitar o suavizar el sesgo presente en los medios de comunicación. No obstante, a pesar de que Factiva incluye una amplia variedad de periódicos, no todos están disponibles durante todo el período analizado, y la cantidad de periódicos incluidos no es la misma en todos los países. Esta restricción de datos supuso una limitación en el período de tiempo y el número de periódicos que utilizamos para elaborar los índices. Si se hubiera utilizado más de un periódico por país, el período de tiempo de los datos habría tenido que reducirse a menos de tres años. Por lo tanto, decidimos seleccionar solo un periódico por país y así extender el marco temporal cubierto por la base de datos, de 2003 a 2016. Como se ha mencionado anteriormente, esta es la principal debilidad de la base de datos, pues los índices podrían estar sesgados como resultado de los diferentes grados de presión ejercida por las industrias extractivas, los gobiernos u otros actores sobre determinados medios de comunicación en lo que respecta a la cobertura de los conflictos en cada país.

Sin embargo, si los efectos de esta presión se mantuvieran constantes a lo largo del tiempo, los índices de la muestra de países solo estarán sesgados en este nivel, lo que no constituye una preocupación real, pues, aun así, su variación proporcionaría información sobre aumentos o reducciones del nivel de conflicto. Surgirían problemas si la intensidad de la presión variara con el tiempo. Si esta variara a lo largo del país, pero se mantuviera estable en el tiempo, se limitaría el análisis a nivel de los países, pero este problema puede resolverse fácilmente mediante la aplicación de un modelo de regresión de efectos fijos. De todos modos, en el futuro deberían tomarse medidas para evitar o minimizar este problema potencial.

Se utilizaron los periódicos de mayor circulación en cada país analizado; a saber: El Mercurio en el caso de Chile (disponible a partir de noviembre de 2002), El Comercio en el del Perú (a partir de octubre de 2002), el Herald Sun en el de Australia (a partir de julio de 1997) y The Globe and Mail en el del Canadá (a partir de diciembre de 1986). La mayor parte de los periódicos contienen artículos referidos a otros países, por lo que eliminamos los artículos que contenían el nombre de otros países y no mencionaban el del país de interés. Se eligió el período que abarca desde 2003 hasta el primer semestre de 2016 para contar con un panel de datos balanceado.

La detección de los conflictos fue una parte crucial del proceso. En consonancia con PNUD/ Fundación UNIR (2012), en este estudio se adopta una definición clásica de conflicto social, entendido como:

un proceso de interacción contenciosa entre actores sociales e instituciones, movilizados con diversos grados de organización y que actúan de manera colectiva de acuerdo con expectativas de mejora, de defensa de la situación preexistente o proponiendo un contraproyecto social. Un conflicto social surge cuando un grupo social, actor o movimiento social (obreros, empresarios, campesinos, indígenas, maestros, movimiento cívico, estudiantes, gremios, universitarios, etc.) expresa una situación de malestar colectivo de forma hostil a través de demandas y medidas de presión violentas (huelgas, marchas, motines, movilizaciones, tomas de instalaciones, paros, revueltas, etc.) contra alguna instancia pública [...] o privada [...]. (pág. 283).

De acuerdo con esta definición, se adoptó un enfoque basado en diccionarios para detectar estos patrones en los artículos de prensa. Se utilizó la categoría de palabras hostiles del diccionario Harvard IV', que contenía 687 entradas en inglés en el momento en que se realizó la consulta. Posteriormente, estas palabras se tradujeron al español y la base de datos se amplió hasta alcanzar un total de 1.326 palabras al añadir los sinónimos de dichas palabras hostiles (véase el anexo A1).

Para evitar cualquier posible complicación que pudiera surgir de la conjugación de los verbos y la concordancia de sustantivos y adjetivos, mediante el programa estadístico R se eliminaron las

\footnotetext{
1 Véase [en línea] http://www.wjh.harvard.edu/ inquirer/homecat.htm.
} 
terminaciones de las palabras más comunes en español y en inglés. A continuación, se reescribieron todas las palabras en minúsculas, se excluyeron la puntuación y las tildes, y se eliminaron las palabras más comunes de cada idioma (como los conectores) para evitar contar palabras adicionales que no añaden significado y pueden ser utilizadas de manera más o menos frecuente en cada idioma.

Por último, el programa R nos permitió sistematizar el proceso de generación de índices para cada país, los cuales se desagregaron posteriormente por área administrativa. Una vez más, se utilizaron técnicas de minería de textos para categorizar los artículos por región, comprobando si contenían el nombre de un área administrativa determinada o de las principales ciudades o explotaciones mineras de dicha área.

Se crearon tres índices alternativos que sirven como variables indirectas de la cantidad de conflictos sociales relacionados con recursos naturales (Palazzo, s/f) y pueden utilizarse con distintos fines:

i) Índice de noticias conflictivas: $\mathrm{Si} C N_{i, t}$ son los artículos de prensa que incluyen palabras hostiles sobre un país o una región $i$ en el momento $t$. El índice de noticias conflictivas para cada país o región es $C N_{i}=\sum_{t} C N_{i, t}$ y el índice total de noticias conflictivas para cada período es $C N_{t}=\sum_{i} C N_{i, t}$. Este índice puede presentar numerosos sesgos, pues, por ejemplo, no tiene en cuenta si un incremento dado del número de palabras hostiles es atribuible únicamente al hecho de que se publiquen más noticias sobre el tema.

ii) Índice de noticias conflictivas estandarizado: De acuerdo con Baker, Bloom y Davis (2013), si $T N_{i, t}$ son los artículos de prensa que tratan sobre las industrias extractivas en un país o región $i$ publicados en el período $t$, el índice de noticias conflictivas estandarizado es $S C N_{i, t}=C N_{i, t} / T N_{i, t}$.

iii) Índice de intensidad del conflicto: Sobre la base de García (2013) y Aromí (2013), medimos la intensidad de un conflicto en un punto específico del tiempo y el espacio como la razón entre el número de palabras hostiles y el número total de palabras del subconjunto de noticias conflictivas. Si $C W_{i, t}$ es el número de palabras hostiles presentes en el índice de noticias conflictivas y $T W_{i, t}$ es el número total de palabras de esos artículos, la intensidad del conflicto en el país o la región $i$ durante el período $t$ es $C I_{i, t}=C W_{i, t} / T W_{i, t}$.

\section{Conflictos sociales relacionados con las actividades extractivas}

\section{Comparación entre países}

Se recopiló un total de 20.119 artículos de prensa sobre industrias extractivas de los cuatro periódicos analizados publicados en el período comprendido entre el primer trimestre de 2003 y el segundo trimestre de 2016. De estos artículos, el 78\% se clasificó como noticias conflictivas (véase el cuadro 1)², lo que indica que el público es, en general, pesimista en lo que se refiere a las actividades extractivas. Esto era esperable, dado que nuestra unidad de análisis son los artículos de prensa.

Cuando los datos se desagregan por país, surgen algunas diferencias. En primer lugar, el porcentaje de noticias conflictivas sobre el total de noticias acerca de las industrias extractivas - capturado por el índice de noticias conflictivas estandarizado $\left(S C N_{i, t}\right)$ - es más elevado en los países desarrollados (en torno al 92\% tanto en el caso de Australia como en el del Canadá), mientras que, en Chile y el Perú, se sitúa entre el $63 \%$ y el $66 \%$. En cuanto a la intensidad del conflicto, los valores son más altos en el caso de los países desarrollados, y la cifra es ligeramente mayor en el del Canadá que en el de Australia.

\footnotetext{
2 Nuestros principales índices, desagregados por mes y año, se pueden consultar en https://sites.google.com/view/gabrielmpalazzo/ original-databases. Los códigos en formato R se encuentran disponibles a solicitud de los interesados.
} 


\section{Cuadro 1}

Australia, Canadá, Chile y Perú: patrones nacionales en los conflictos sociales relacionados con actividades extractivas, primer trimestre de 2003 a segundo trimestre de 2016

(En número total de artículos y porcentajes)

\begin{tabular}{lcccc}
\hline País & $\begin{array}{c}\text { Número total de artículos } \\
\text { sobre actividades } \\
\text { extractivas } \\
T N_{i, t}\end{array}$ & $\begin{array}{c}\text { Noticias conflictivas } \\
C N_{i, t}\end{array}$ & $\begin{array}{c}\text { Noticias conflictivas } \\
\text { sobre el total de artículos } \\
\text { (índice estandarizado) } \\
\begin{array}{c}S C N_{i, t} \\
\text { (en porcentajes) }\end{array}\end{array}$ & $\begin{array}{c}\text { Intensidad del conflicto } \\
C I_{i, t} \\
\text { (en porcentajes) }\end{array}$ \\
\hline Australia & 2709 & 2502 & 92,36 & 3,70 \\
\hline Canadá & 6871 & 6349 & 92,40 & 4,53 \\
\hline Chile & 8095 & 5375 & 66,40 & 2,76 \\
\hline Perú & 2444 & 1543 & 63,13 & 2,89 \\
\hline Total & 20119 & 15769 & 78,37 & 3,81 \\
\hline
\end{tabular}

Fuente: Elaboración propia, sobre la base de artículos obtenidos de los periódicos El Mercurio, Comercio, The Globe and Mail y Herald Sun.

Estos resultados describen en términos generales los resultados del presente trabajo y la base de datos que hemos elaborado. No obstante, no debe interpretarse que los niveles de conflicto en Australia y el Canadá son más elevados que en Chile y el Perú. En primer lugar, puesto que comparamos índices creados a partir de distintos periódicos, una proporción más elevada de noticias conflictivas sobre la cantidad total de noticias $\left(S C N_{i, t}\right)$ o una mayor intensidad del conflicto $\left(C I_{i, t}\right)$ podría explicarse por las preferencias o intereses de los lectores de cada publicación, así como por los diferentes estilos de escritura de los periodistas. Por otro lado, las diferencias de nivel entre los países analizados coinciden con las diferencias lingüísticas, por lo que no puede descartarse el hecho de que los hablantes nativos de las dos lenguas expresen los hechos y sus opiniones de maneras distintas. Por último, como se mencionó anteriormente, las diferencias pueden atribuirse a diferentes sesgos de los medios de comunicación. Estos resultados, por lo tanto, deben interpretarse como índices compuestos por distintas constantes y el análisis debe centrarse en la manera en que el comportamiento se modifica a lo largo del tiempo y en las respuestas que surgen ante choques relevantes provenientes de variables exógenas.

A continuación, nos centraremos en los patrones de tiempo (véase el cuadro 2). Es posible detectar tres subperíodos diferenciados en lo que respecta al índice de noticias conflictivas, $C N_{i, t}$. El primero se extiende aproximadamente desde 2003 hasta 2006, cuando los conflictos sociales parecen incrementarse; el segundo, desde 2007 hasta 2009, cuando los conflictos disminuyen lentamente, y el tercero, desde 2010 hasta 2016, cuando, por lo general, se mantienen estables, a excepción de dos picos, uno en 2010 y otro en 2014. Sin embargo, el índice estandarizado $S C N_{i, t}$ revela un panorama distinto: la tendencia fue relativamente estable durante el período 2003-2007, lo que refleja que las noticias conflictivas aumentaron al mismo ritmo que el número total de noticias, antes de dar un salto y estabilizarse en niveles superiores durante el período 2008-2013. Por último, el nivel fluctúa a lo largo del período 2014-2016 y alcanza su máximo en 2015.

En lo referente a la intensidad del conflicto, el índice $C I_{i, t}$ muestra que la intensidad de los conflictos sociales relacionados con actividades extractivas aumentó de manera constante entre 2003 y 2010, antes de comenzar a disminuir lentamente.

Por último, el gráfico 1 muestra la evolución de los conflictos sociales a lo largo del tiempo en cada país. Es evidente que tanto el índice de noticias totales $T N_{i, t}$ como el índice de noticias conflictivas $C N_{i, t}$ alcanzaron su nivel máximo en el período 2004-2008. A su vez, el índice estandarizado revela que las diferencias entre países mencionadas anteriormente se encuentran presentes en todo el período analizado. Los índices de Chile y el Perú son más volátiles que los de Australia y el Canadá, y los conflictos sociales en Chile aumentaron significativamente durante los últimos dos años del período analizado. Con respecto a la intensidad del conflicto, pueden hacerse dos observaciones interesantes. 
En primer lugar, que el índice del Perú es más volátil que los demás, lo que significa que, a diferencia de los otros países, experimenta "explosiones" de conflictos intensos. En segundo lugar, que la intensidad del conflicto se mantuvo relativamente baja en Chile a lo largo de todo el período ${ }^{3}$.

\section{Cuadro 2}

Australia, Canadá, Chile y Perú: patrones de tiempo en los conflictos sociales relacionados con actividades extractivas, 2003-2016

(En número total de artículos y porcentajes)

\begin{tabular}{lcccc}
\hline Año $t$ & $\begin{array}{c}\text { Número total de artículos } \\
\text { sobre actividades extractivas } \\
T N_{i, t}\end{array}$ & $\begin{array}{c}\text { Noticias conflictivas } \\
C N_{i, t}\end{array}$ & $\begin{array}{c}\text { Noticias conflictivas } \\
\text { sobre el total de artículos } \\
\text { (indice estandarizado) } \\
\text { SCN } N_{i, t} \\
\text { (en porcentajes) }\end{array}$ & $\begin{array}{c}\text { Intensidad del conflicto } \\
C_{i, t} \\
\text { (en porcentajes) }\end{array}$ \\
\hline 2003 & 822 & 695 & 84,55 & 4,02 \\
\hline 2004 & 1394 & 1049 & 75,25 & 3,42 \\
\hline 2005 & 1497 & 1091 & 72,88 & 3,20 \\
\hline 2006 & 2128 & 1637 & 76,93 & 3,62 \\
\hline 2007 & 1952 & 1270 & 65,06 & 3,63 \\
\hline 2008 & 953 & 664 & 69,67 & 3,47 \\
\hline 2010 & 774 & 529 & 68,35 & 4,02 \\
\hline 2011 & 938 & 755 & 80,49 & 4,42 \\
\hline 2012 & 670 & 495 & 73,88 & 4,03 \\
\hline 2013 & 669 & 541 & 80,87 & 4,03 \\
\hline 2015 & 732 & 535 & 73,09 & 3,98 \\
\hline 2016 & 908 & 502 & 55,29 & 3,75 \\
\hline
\end{tabular}

Fuente: Elaboración propia, sobre la base de artículos obtenidos de los periódicos El Mercurio, Comercio, The Globe and Mail y Herald Sun.

La ventaja principal de la técnica de la minería de textos es que permite procesar una enorme cantidad de datos desestructurados sin necesidad de realizar un análisis cualitativo. No obstante, podría resultar informativo establecer correspondencias entre algunos de los picos y determinados sucesos relevantes. Por ejemplo, en noviembre de 2010, hubo un pico en las noticias conflictivas en el Canadá, que coincidió con el controvertido intento de la empresa australiana BHP Billiton de adquirir PotashCorp, bloqueado por el Primer Ministro del Canadá, Sthephen Harper. Este acontecimiento se ve reflejado en el gráfico 1, en el aumento de las noticias conflictivas durante 2010 en el Canadá. Otro ejemplo es una huelga organizada en Chile, en diciembre de 2005, por los empleados del Ferrocarril de Antofagasta a Bolivia (FCAB) - una línea de ferrocarril para la industria minera de propiedad privada que va de Antofagasta, en el norte de Chile, al Estado Plurinacional de Bolivia- con el objetivo de conseguir un aumento salarial. En ese momento existía también cierta polémica por la apreciación del peso chileno respecto del dólar, y las empresas mineras se quejaban del efecto que esto tenía en sus ganancias. Además, durante los primeros meses de 2006, algunos miembros de la oposición plantearon preguntas en el Parlamento acerca de la contratación de consultores y servicios de la Corporación Nacional del Cobre Chileno (CODELCO), una empresa estatal chilena dedicada a la minería del cobre. Todos estos sucesos coinciden con el punto máximo registrado en el índice de noticias conflictivas de Chile en 2006.

\footnotetext{
3 Debe tenerse en cuenta que, aunque los conflictos surjan en un territorio específico, es posible que empresas de otros países sean partícipes de ellos. Por ejemplo, algunas empresas canadienses podrían estar involucradas en conflictos surgidos en África o en Chile. Mediante la metodología utilizada en este trabajo, no es posible resolver esta dificultad relacionada con los datos; si la noticia aparece en un periódico canadiense, se clasificará como una noticia conflictiva perteneciente a ese país.
} 


\section{Gráfico 1}

Australia, Canadá, Chile y Perú: conflictos sociales relacionados con actividades extractivas, 2003-2016

A. Total de noticias (en número total de artículos)

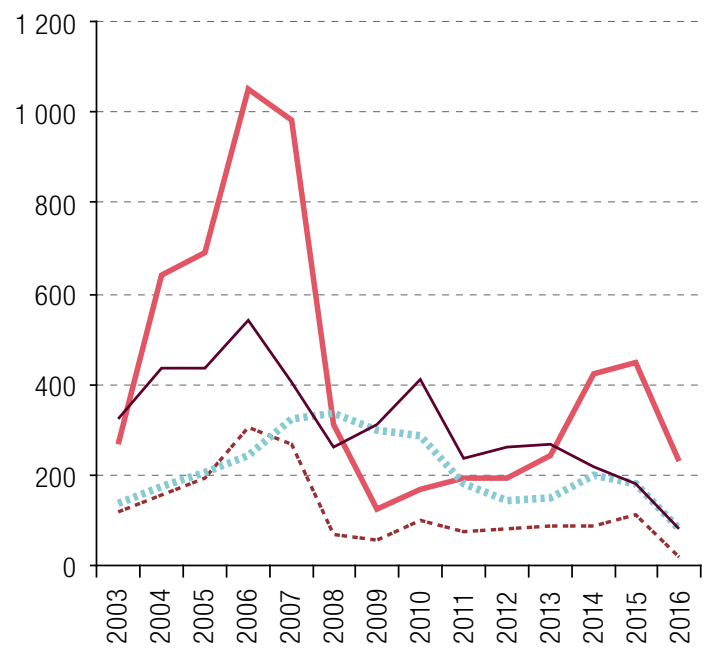

C. Noticias conflictivas sobre el total de artículos (índice estandarizado) (en porcentajes)

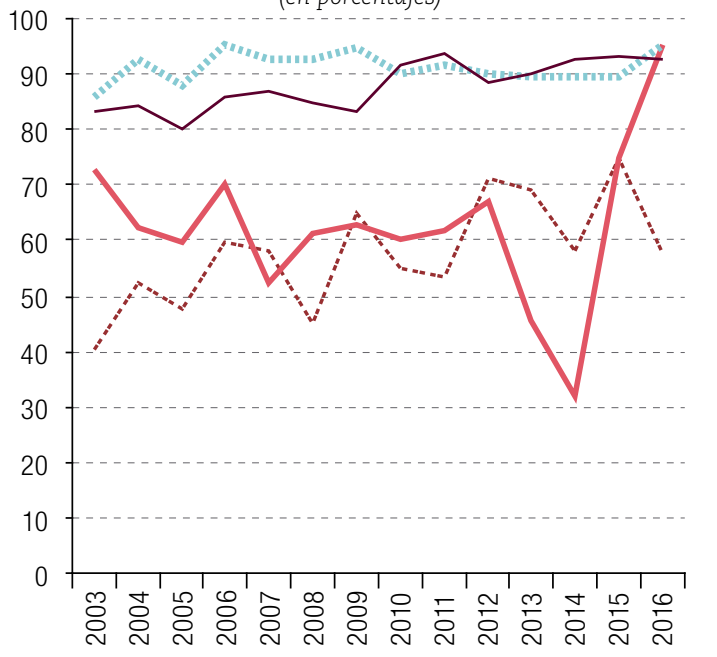

B. Noticias conflictivas (en número total de artículos)

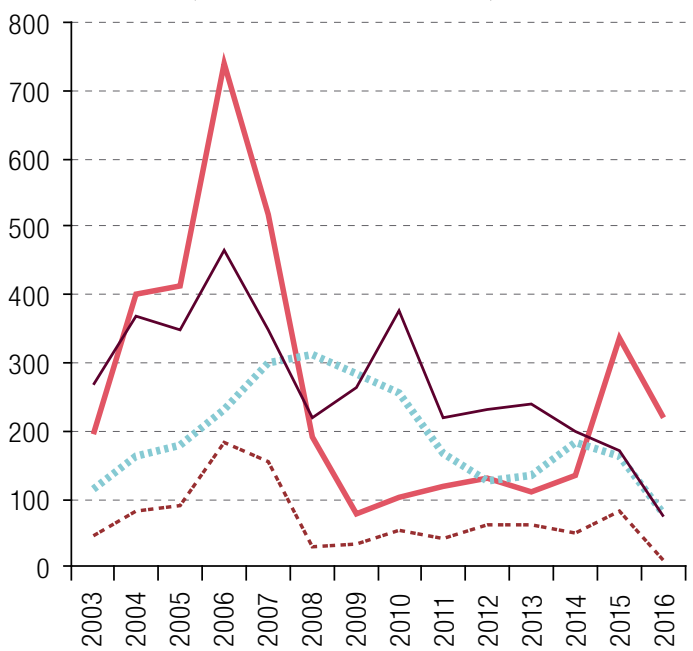
(en porcentajes)

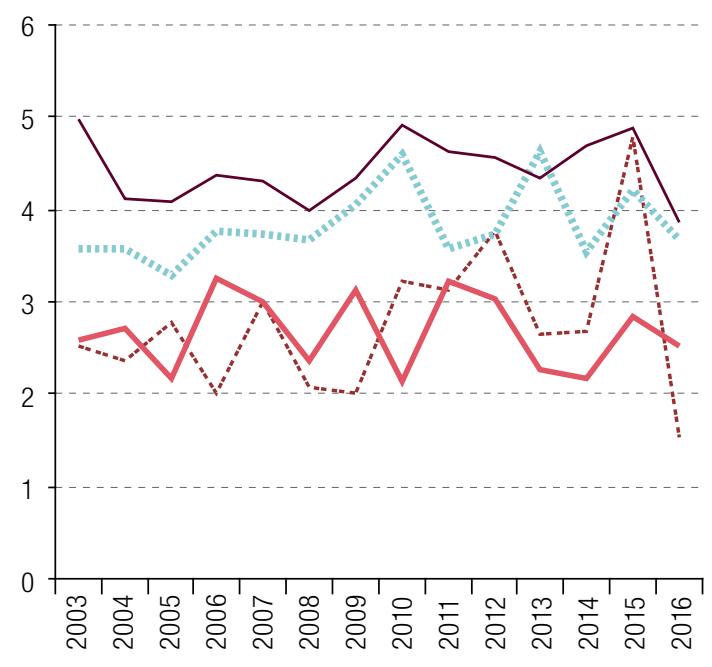

- Australia - Canadá

Estos son ejemplos del tipo de conflictos que capturan los índices, pero no contamos con una medida objetiva de conflictos para comprobar su precisión. Tratar de comprobar la precisión de los índices contrastándolos con los artículos de prensa no sería concluyente, dado que estos son la fuente de información primaria para la elaboración de los índices. Aun así, los índices son un primer paso para documentar los conflictos civiles relacionados con las actividades extractivas en el futuro.

\section{Comparaciones regionales}

A fin de desagregar los conflictos por área administrativa, utilizamos el enfoque de la minería de textos para clasificar cada artículo por estado, territorio, provincia, región o departamento, determinando si incluían el nombre del área administrativa en cuestión, de las capitales o de las explotaciones mineras de dicha área. 
Como era de esperar, las áreas administrativas en las que se registró una mayor participación del total de artículos de prensa relacionados con la minería fueron aquellas en las que se encuentran presentes las industrias extractivas. En el gráfico 2 se muestra la relación entre la participación de cada área administrativa en el producto bruto interno (PBI) de la minería a nivel nacional alrededor de 2013 y 2014, y su participación en el total de artículos de prensa sobre minería de cada país.

\section{Gráfico 2}

Australia, Canadá, Chile y Perú: participación en las actividades extractivas y en los artículos de prensa sobre minería, por área administrativa, primer trimestre de 2003 a segundo trimestre de 2016
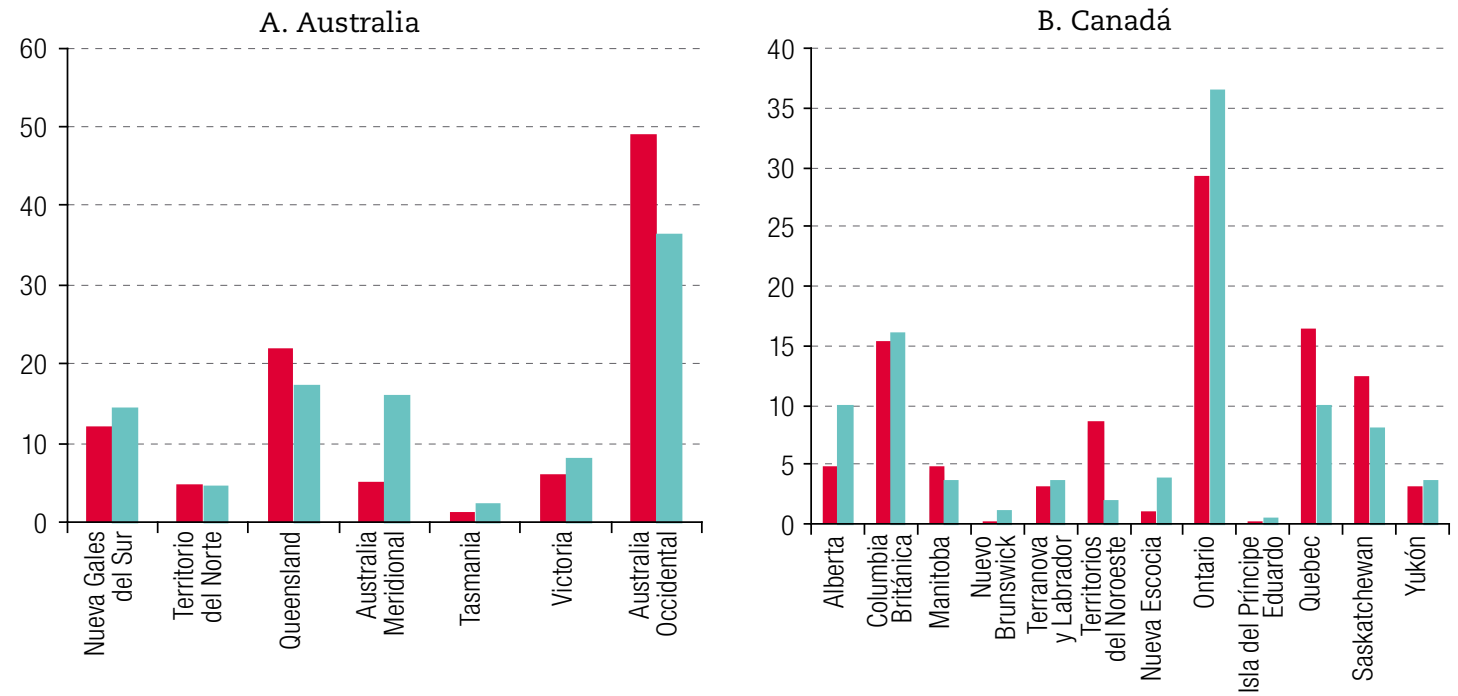

C. Chile

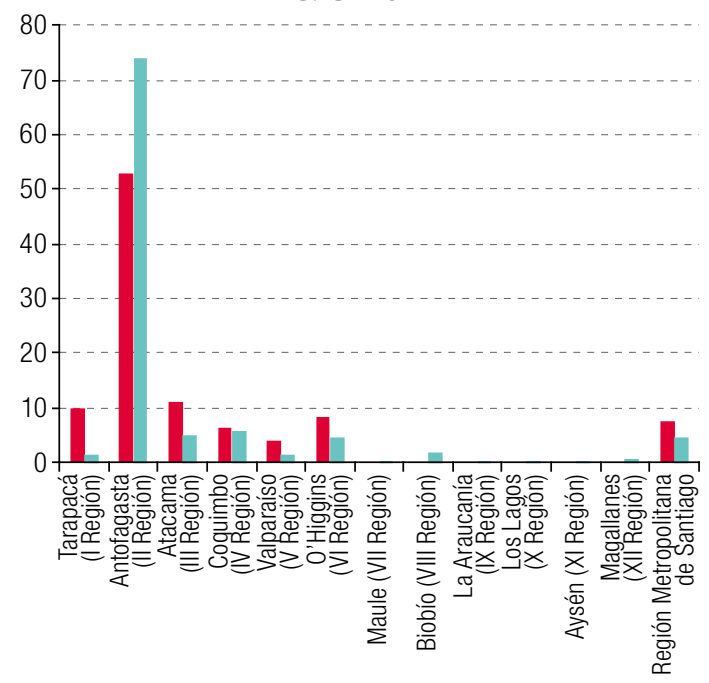

D. Perú

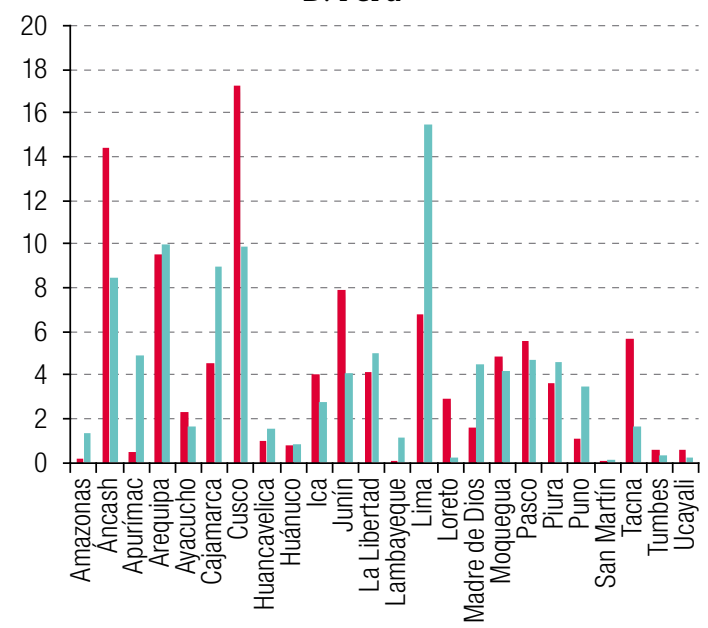

Participación en la actividad extractiva

Participación en el total de noticias

Fuente: Elaboración propia.

Nota: No se encontraron datos sobre las áreas administrativas del Territorio de la Capital de Australia (Australia); Nunavut (Canadá); Los Ríos (XIV Región), Arica y Parinacota (XV Región) y Ñuble (XVI Región) (Chile), y Callao (Perú), por lo que no se incluyen en estos índices. 
Los resultados indican que existe una clara correlación entre ambos índices, especialmente en el caso de Ontario (Canadá), Australia Occidental (Australia) y Antofagasta (II Región) (Chile), pues las principales regiones productoras presentan la mayor cantidad de artículos de prensa sobre la minería. El número de artículos disminuye a medida que el sector minero se vuelve menos importante.

Esta correlación es más débil en el caso del Perú, si bien se mantiene elevada. Lima presenta la participación más alta de artículos de prensa sobre la minería, pero se sitúa en el quinto lugar en lo que se refiere a actividad extractiva (7\%). Aunque no es el principal departamento minero (Cusco y Áncash son los más importantes), es lógico que se informe sobre noticias relacionadas con la minería en la capital, donde están los políticos nacionales y los parlamentarios. Estos resultados coinciden con los de Palazzo (2017).

Para obtener los valores regionales del índice de noticias conflictivas estandarizado y el índice de intensidad del conflicto, se elaboraron mapas regionales de conflictos sociales relacionados con la industria extractiva. A fin de analizar las diferencias regionales dentro de cada país, se consideraron cuatro subgrupos distintos: considerablemente superior a la media, superior a la media, inferior a la media y considerablemente inferior a la media. Los dos subgrupos que se encuentran considerablemente por encima o por debajo de la media se refieren a los casos en los que el índice alcanza valores superiores o inferiores al valor medio más (o menos) una desviación estándar. Los otros dos subgrupos exhiben valores superiores o inferiores a la media, pero dentro del rango normal de desviación (véase el mapa 1).

En lo que respecta al índice de noticias conflictivas estandarizado, en Australia, Queensland presenta un nivel de conflicto particularmente elevado. Por ejemplo, la proporción de noticias negativas supera el 95\% (el 92,3\% es el promedio del país en conjunto), mientras que Australia Occidental y el Territorio del Norte presentan niveles de conflicto relativamente bajos (alrededor del $90 \%$ de los artículos de prensa contienen palabras hostiles). La variabilidad total, sin embargo, es relativamente baja (el coeficiente de variación es de 0,023).

En el Canadá, el índice de noticias conflictivas estandarizado también se acerca al 92\%. Nuevo Brunswick y Terranova, dos provincias del este, destacan por su alta proporción de noticias negativas, superior al 95\%, mientras que Nueva Escocia presenta la más baja (el 84,5\% de los artículos de prensa contienen palabras hostiles). Este valor atípico significa que el índice de variabilidad total del Canadá es superior al de Australia.

Chile tiene un nivel de conflicto menor: su índice está en torno al 80\%. Las diferencias entre regiones están más marcadas que en los otros casos, por lo que su coeficiente de variación es más elevado $(0,12)$. El índice supera el $85 \%$ en algunas regiones, mientras que en otras es inferior al $70 \%$. En el primer grupo se incluyen tres regiones que no son áreas mineras tradicionales: Aysén (XI Región), Los Lagos (X Región) y Magallanes (XII Región). Es interesante subrayar que una de las principales áreas mineras de Chile, Antofagasta (II Región), está entre las regiones -junto con La Araucanía (IX Región) - con los niveles más bajos de conflicto (con solo un 61\% de artículos de prensa negativos).

En el Perú, por último, el contraste entre los diferentes departamentos es todavía más marcado: algunos presentan un índice de noticias conflictivas estandarizado inferior al 50\%, mientras que, en otros, este se acerca al 90\% (el coeficiente de variación asciende a 0,23). Tres departamentos son especialmente propensos al conflicto - Amazonas, Madre de Dios y Tumbes -, mientras que Ucayali tiene el menor nivel de conflictos asociados a la actividad extractiva. 


\section{Mapa 1}

Australia, Canadá, Chile y Perú: índice de noticias conflictivas estandardizado por área administrativa, primer trimestre de 2003 a segundo trimestre de 2016

A. Australia

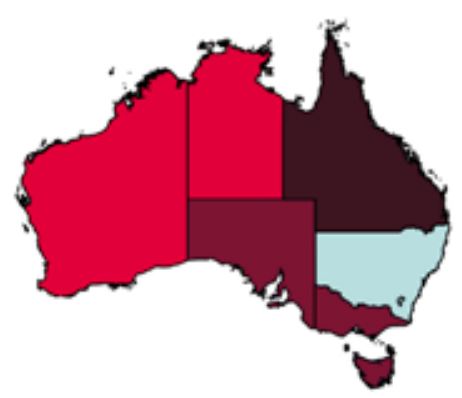

C. Chile

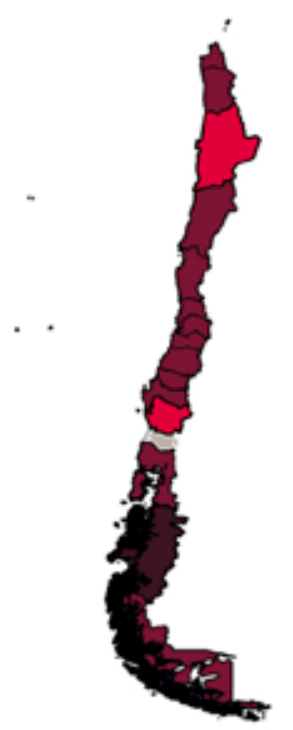

B. Canadá

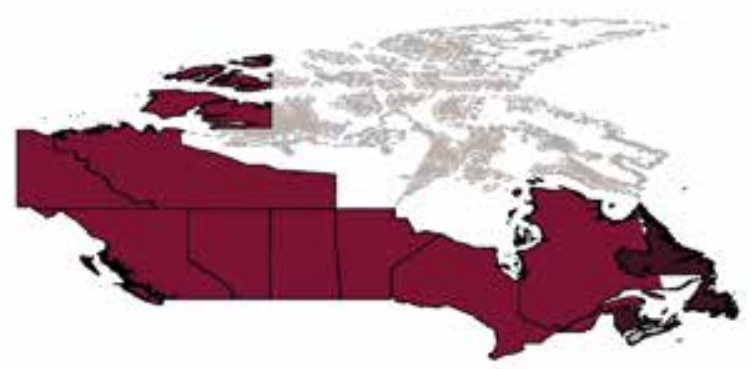

D. Perú

Considerablemente inferior a la media Considerablemente superior a la media

- Superior a la media Inferior a la media Sin datos

Fuente: Elaboración propia.

Nota: No se encontraron datos sobre las áreas administrativas del Territorio de la Capital de Australia (Australia); Nunavut (Canadá); Los Ríos (XIV Región), Arica y Parinacota (XV Región) y Ñuble (XVI Región) (Chile), y Callao (Perú), por lo que no se incluyen en estos índices.

En cuanto al índice de intensidad del conflicto (véase el mapa 2), Victoria es el estado australiano con la mayor intensidad: casi 1 de cada 20 palabras contenidas en los artículos de prensa relevantes es hostil. Por su parte, Queensland, que presenta el nivel de conflicto más elevado, registra un nivel más bajo de intensidad del conflicto, como esperábamos. Por otro lado, el Territorio del Norte cuenta tanto con el nivel más bajo de conflicto como con la razón más baja de intensidad (1 de cada 30 palabras es hostil). 


\section{Mapa 2}

Australia, Canadá, Chile y Perú: índice de intensidad del conflicto por área administrativa, primer trimestre de 2003 a segundo trimestre de 2016

A. Australia

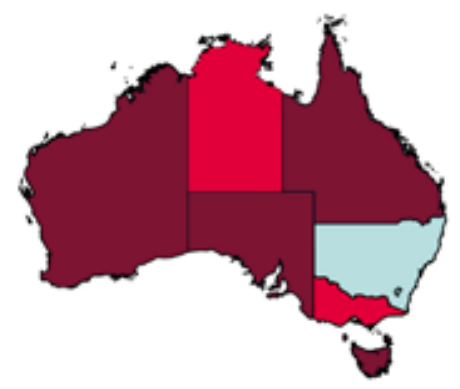

C. Chile

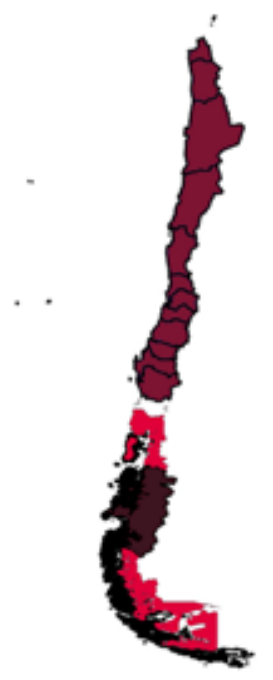

B. Canadá

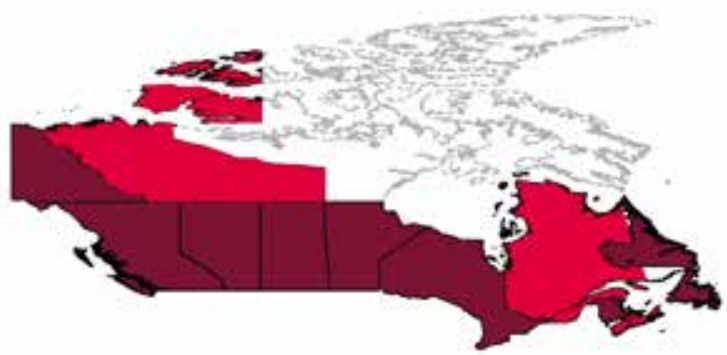

D. Perú

Superior a la media Considerablemente superior a la media Inferior a la media

- Considerablemente inferior a la media Sin datos

Fuente: Elaboración propia.

Nota: $\quad$ No se encontraron datos sobre las áreas administrativas del Territorio de la Capital de Australia (Australia); Nunavut (Canadá); Los Ríos (XIV Región), Arica y Parinacota (XV Región) y Ñuble (XVI Región) (Chile), y Callao (Perú), por lo que no se incluyen en estos índices.

La intensidad del conflicto es mucho más homogénea en las áreas administrativas del Canadá: el coeficiente de variación es de 0,045 y el porcentaje de palabras hostiles varía entre el 4,0\% y el 4,7\%. Dos áreas destacan por su baja intensidad del conflicto: los Territorios del Noroeste y Nueva Escocia.

Las disparidades en la intensidad de los conflictos sociales son más pronunciadas en los países en desarrollo. En Chile, por ejemplo, las regiones de Aysén (XI Región), Maule (VII Región) y O'Higgins (VI Región) registran niveles de intensidad del conflicto elevados, mientras que los niveles de Magallanes (XII Región) y Los Lagos (X Región) son los menores. A causa de estas diferencias, el coeficiente de variación es de 0,20. 
Las diferencias son incluso mayores en el Perú. En cuatro departamentos (Madre de Dios, Huánuco, Puno y Amazonas), la intensidad del conflicto es elevada, dado que al menos 1 de cada 30 palabras está relacionada con algún conflicto, mientras que es baja en otros cuatro (Junín, La Libertad, Lambayeque y Tumbes). El coeficiente de variación del Perú $(0,25)$ es el más alto de los cuatro países analizados.

\section{Intensidad de la violencia de los conflictos}

Puede parecer contrario a la lógica que los resultados de los índices de conflicto social relacionados con las actividades extractivas sean más elevados en los países desarrollados que en los países en desarrollo. Si bien ya se han proporcionado algunas posibles razones metodológicas de este hecho y se ha explicado la mejor manera de utilizar los índices, a continuación se presentan algunas posibles explicaciones para los distintos tipos de conflicto que captan los índices en la muestra de cada país. Una hipótesis comprobable es que los índices de los países desarrollados captan conflictos menos violentos que los de los países en desarrollo. Los índices están construidos de tal manera que, por ejemplo, la palabra "muerte" tiene el mismo peso que la palabra "desacuerdo". La intensidad del conflicto debe redefinirse, por lo tanto, para permitirnos discriminar entre conflictos violentos y no violentos.

Con ese objetivo, elaboramos un índice de conflictos violentos basado en la proporción de palabras hostiles en un momento y un país determinados que pueden clasificarse como violentas; es decir, $V C_{i, t}=V W_{i, t} / C W_{i, t}$, donde $V C_{i, t}$ es el índice y $V W_{i, t}$ es la suma de palabras violentas (el subgrupo de palabras hostiles que son violentas puede consultarse en el anexo A1). $C W_{i, t}$ es la suma de las palabras hostiles definidas más arriba. En el gráfico 3 se presentan los resultados.

Gráfico 3

Australia, Canadá, Chile y Perú: conflictos violentos relacionados con actividades extractivas, 2003-2016

(En porcentajes)

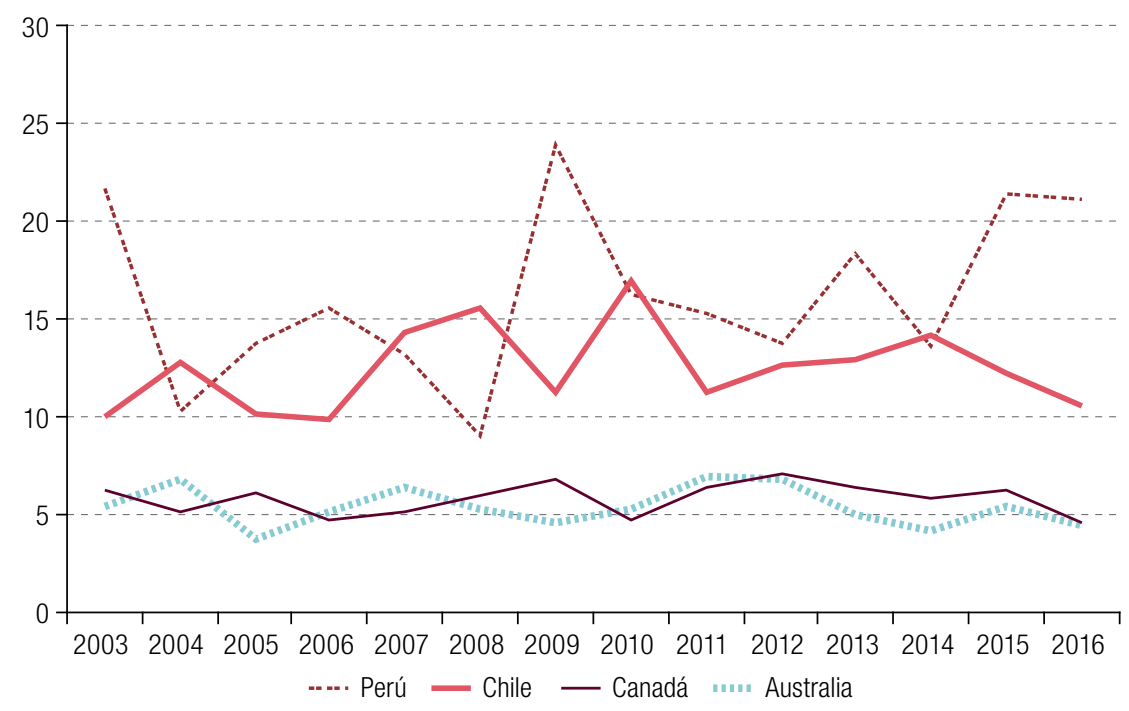

Fuente: Elaboración propia.

Como era de suponer, los conflictos violentos relacionados con actividades extractivas resultaron más habituales en los países en desarrollo. En Australia y el Canadá, la proporción de conflictos violentos en el total de los conflictos hostiles fue en todos los casos cercana al 5\%. En el caso de Chile, el análisis arrojó un promedio del 11\% para todo el período 2003-2016, mientras que, en el Perú, el promedio se 
situó en torno al 15\%. Nuestra principal hipótesis es que estos resultados pueden tener que ver con factores como una gobernanza más débil o la menor disponibilidad de mecanismos para enfrentar y resolver las demandas sociales en los países en desarrollo, lo que se traduciría en oleadas de conflictos violentos. Además, en estos países, la desigualdad es mayor y la polarización, más pronunciada, por lo que los conflictos civiles son más violentos que en los países desarrollados.

También debe tenerse en cuenta que algunos conflictos menos violentos en los países en desarrollo podrían recibir una menor cobertura mediática gracias a la mayor influencia que ejercen las élites - incluidos los propietarios de los yacimientos mineros - para suavizar las noticias desfavorables (véanse análisis más detallados de esta hipótesis en Nafziger y Auvinen (2002) y Sinnott, Nash y De la Torre (2010)). Sin embargo, es necesario llevar a cabo más estudios y contar con una muestra más amplia de países para terminar de comprender esta diferencia entre los países desarrollados y los países en desarrollo.

\section{Algunos hechos estilizados de la base de datos}

\section{1. ¿Factores comunes o tendencias específicas de cada país?}

A partir de un análisis más exhaustivo de la correlación entre los índices de conflicto de nuestra muestra, se obtienen algunos resultados interesantes. El cuadro 3 contiene la matriz de correlaciones, en la que se utiliza la frecuencia anual de los diferentes índices de conflictos.

Cuadro 3

Australia, Canadá, Chile y Perú: matriz de correlación

\begin{tabular}{|c|c|c|c|c|c|c|c|c|}
\hline \multirow[b]{2}{*}{ País } & \multicolumn{4}{|c|}{ A. Noticias conflictivas } & \multicolumn{4}{|c|}{$\begin{array}{l}\text { B. Noticias conflictivas sobre el total de artículos } \\
\text { (índice estandarizado) }\end{array}$} \\
\hline & Chile & Perú & Canadá & Australia & Chile & Perú & Canadá & Australia \\
\hline Chile & 1 & & & & 1 & & & \\
\hline Perú & 0,9337 & 1 & & & $-0,023$ & 1 & & \\
\hline Canadá & 0,6876 & 0,6976 & 1 & & $-0,2708$ & 0,514 & 1 & \\
\hline \multirow[t]{2}{*}{ Australia } & 0,1821 & 0,1894 & 0,2793 & 1 & 0,0544 & 0,1963 & $-0,056$ & 1 \\
\hline & \multicolumn{4}{|c|}{ C. Intensidad del conflicto } & \multicolumn{4}{|c|}{ D. Intensidad del conflicto violento } \\
\hline País & Chile & Perú & Canadá & Australia & Chile & Perú & Canadá & Australia \\
\hline Chile & 1 & & & & 1 & & & \\
\hline Perú & 0,0559 & 1 & & & $-0,4069$ & 1 & & \\
\hline Canadá & 0,0165 & 0,5365 & 1 & & $-0,3009$ & 0,3782 & 1 & \\
\hline Australia & $-0,1647$ & 0,2451 & 0,3076 & 1 & 0,0878 & $-0,2599$ & $-0,024$ & 1 \\
\hline
\end{tabular}

Fuente: Elaboración propia.

El análisis de las correlaciones llevado a cabo sobre la base datos anuales muestra que, sin ningún tipo de control, los conflictos relacionados con actividades extractivas en los países de la muestra no covarían. Solo muestran alguna correlación positiva y significativa las noticias conflictivas de Chile, el Perú y el Canadá. Australia no se correlaciona de manera positiva y estadísticamente significativa con ninguno de ellos. Los resultados se confirmaron mediante el uso de datos trimestrales y la realización de un análisis factorial (véase el cuadro 4$)^{4}$.

\footnotetext{
4 Se excluyeron del índice de intensidad del conflicto de Chile ocho observaciones mensuales cuyos valores equivalían a cinco veces la desviación estándar por encima de la media. Estos valores extremos solo estaban presentes en el caso de Chile y podrían haber introducido sesgos en el análisis de la matriz de correlaciones.
} 
Cuadro 4

Australia, Canadá, Chile y Perú: cargas factoriales ${ }^{a}$ y varianzas únicas

\begin{tabular}{|c|c|c|c|c|c|c|}
\hline \multirow[b]{2}{*}{ País } & \multicolumn{3}{|c|}{ A. Noticias conflictivas } & \multicolumn{3}{|c|}{$\begin{array}{l}\text { B. Noticias conflictivas sobre el total de artículos } \\
\text { (indice estandarizado) }\end{array}$} \\
\hline & Factor 1 & Factor 2 & Unicidad & Factor 1 & Factor 2 & Unicidad \\
\hline Chile & 0,8047 & $-0,0213$ & 0,352 & $-0,2342$ & 0,3098 & 0,8491 \\
\hline Perú & 0,7634 & 0,0028 & 0,4172 & 0,2368 & 0,3054 & 0,8506 \\
\hline Canadá & 0,565 & 0,0043 & 0,6807 & 0,4341 & $-0,0043$ & 0,8115 \\
\hline \multirow[t]{2}{*}{ Australia } & 0,1375 & 0,0916 & 0,9727 & 0,0658 & 0,0323 & 0,9946 \\
\hline & \multicolumn{3}{|c|}{ C. Intensidad del conflicto } & \multicolumn{3}{|c|}{ D. Intensidad del conflicto violento } \\
\hline País & Factor 1 & Factor 2 & Unicidad & Factor 1 & Factor 2 & Unicidad \\
\hline Chile & 0,5141 & $-0,096$ & 0,7265 & $-0,098$ & 0,1821 & 0,9572 \\
\hline Perú & 0,505 & 0,0926 & 0,7364 & 0,28 & $-0,1119$ & 0,9091 \\
\hline Canadá & 0,2574 & 0,0478 & 0,9315 & 0,3582 & 0,1113 & 0,8593 \\
\hline Australia & $-0,0343$ & 0,2837 & 0,9183 & $-0,4298$ & $-0,0217$ & 0,8148 \\
\hline
\end{tabular}

Fuente: Elaboración propia.

a Las cargas factoriales representan, por un lado, cómo se ponderan las variables para cada factor y, por otro, la correlación entre las variables y el factor.

La unicidad muestra la proporción de la varianza común de la variable que no está asociada a los factores. La cifra correspondiente a la unicidad de las noticias conflictivas es elevada en todos los países, pero sobre todo en Australia. Las cifras de unicidad de los índices de noticias conflictivas estandarizado, intensidad del conflicto e intensidad del conflicto violento también son muy elevadas, con un promedio de $0,876,0,828$ y 0,885 , respectivamente. Estos resultados podrían estar indicando que los conflictos relacionados con las actividades extractivas surgen debido a situaciones políticas y económicas locales y que los diferentes países gestionan los choques comunes de maneras muy diferentes, dependiendo de sus marcos institucionales, su industria y sus estructuras sociales generales. En el gráfico 4 se incluyen el factor principal de los índices y un índice de precio de los metales básicos, extraído de la base de datos International Financial Statistics (IFS), del Fondo Monetario Internacional (FMI).

Del gráfico 4 no se pueden extraer conclusiones evidentes, y debe tenerse en cuenta que la unicidad de cada índice es muy elevada. Sin embargo, el gráfico sí indica que los índices de intensidad del conflicto y de intensidad del conflicto violento (véanse los subgráficos 4C y 4D) no están relacionados con fluctuaciones del índice de precios de los metales básicos, por lo que no se llevó a cabo un análisis econométrico de dichos índices.

En lo que respecta a la evolución de las noticias conflictivas (véase el subgráfico 4A), se observa un aumento tanto en los precios de los metales como en las noticias conflictivas hasta 2007, pero, posteriormente, las tendencias se bifurcan, pues los precios de los metales básicos experimentan una marcada subida en 2008. Una posible explicación de esta divergencia podría ser que, en esa época, los medios de comunicación de los países de nuestra muestra se centraron en la crisis financiera internacional. Otra razón podría ser que el número total de conflictos relacionados con actividades extractivas se volvió un rasgo más específico de cada país tras la crisis.

Si la primera explicación fuera correcta, este problema podría solucionarse utilizando el índice de noticias conflictivas estandarizado, cuyo objetivo es corregir el número total de artículos de prensa sobre conflictos dividiéndolo por el total de artículos de prensa relacionados con actividades extractivas. El factor principal del índice de noticias conflictivas estandarizado (véase el subgráfico 4B) describe una trayectoria ascendente durante todo el período analizado. La relación positiva entre las dos series podría ser espuria si ambas series son I(1). 


\section{Gráfico 4}

Australia, Canadá, Chile y Perú: precios de los metales básicosa y carga factorial principal, primer trimestre de 2003 a cuarto trimestre de 2015

A. Noticias conflictivas

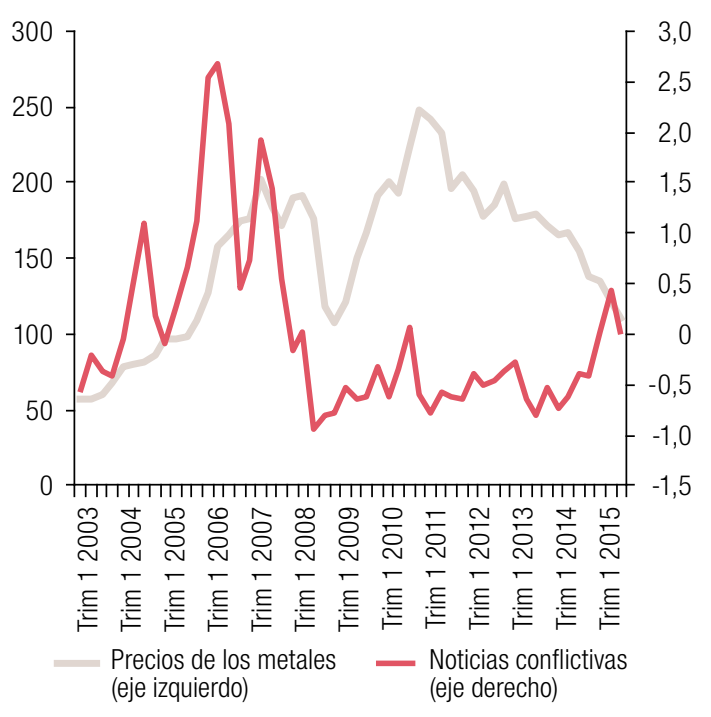

C. Intensidad del conflicto

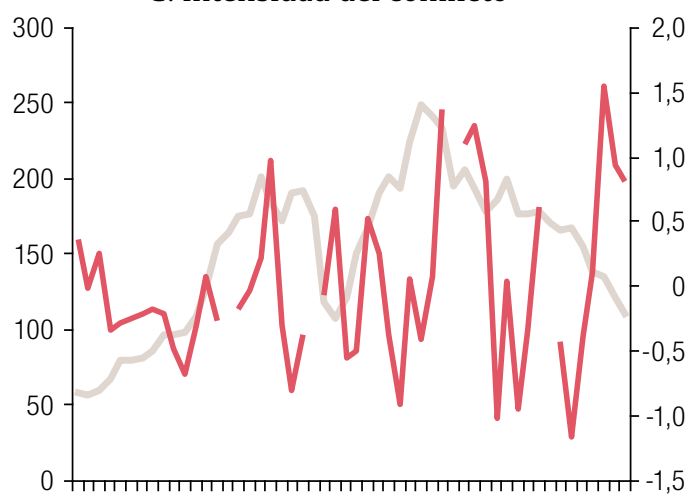

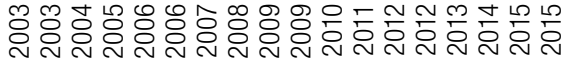

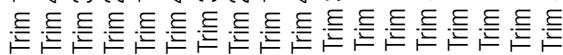

Precios de los metales — Intensidad del conflicto (eje izquierdo)
B. Noticias conflictivas sobre el total de artículos (indice estandarizado)

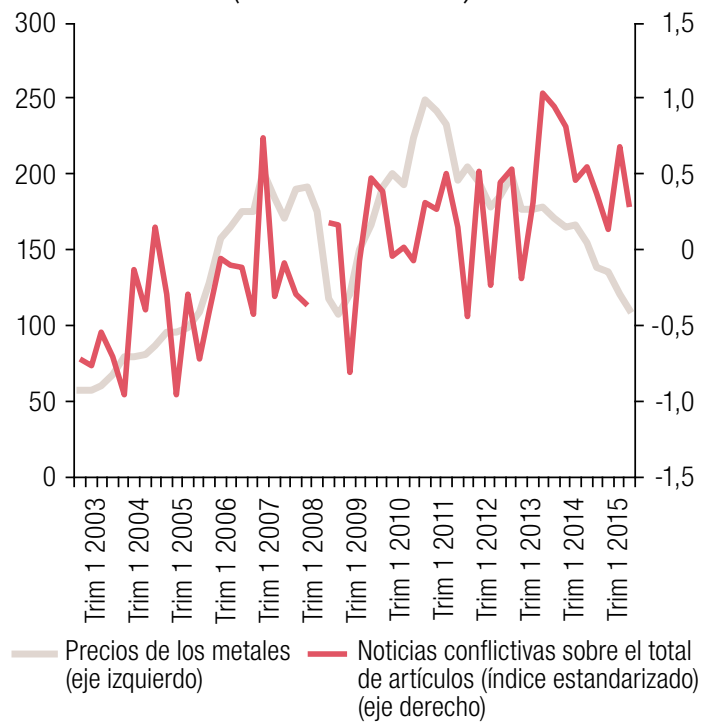

D. Intensidad del conflicto violento

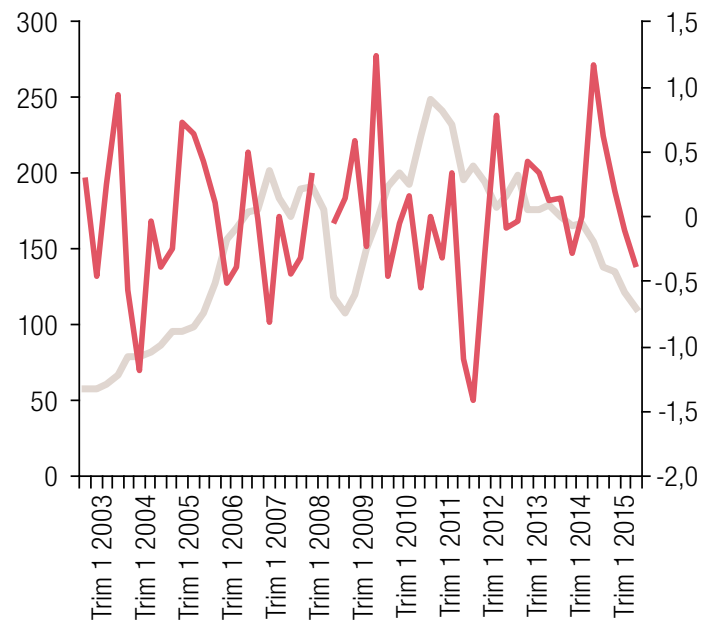

Precios de los metales _ Intensidad del conflicto violento (eje izquierdo) (eje derecho)

Fuente: Elaboración propia, sobre la base de Fondo Monetario Internacional (FMI), "International Financial Statistics (IFS)", 2020 [base de datos en línea] https://data.imf.org/?sk=4C514D48-B6BA-49ED-8AB9-52B0C1A0179B\&sld=1409151240976.

a Índice de precios de los metales básicos, $2005=100$. Incluye los índices de precios del cobre, el aluminio, el mineral de hierro, el estaño, el níquel, el cinc, el plomo y el uranio.

Por ese motivo, se llevó a cabo una prueba de Dickey-Fuller aumentada. La hipótesis nula es que la variable contiene una raíz unitaria y la alternativa es que la variable fue generada por un proceso estacionario. Dado que ambas series muestran un proceso de raíz unitaria ${ }^{5}$, pero la primera diferencia es estacionaria, probamos una relación de cointegración usando la metodología de Engle y Granger ${ }^{6}$.

\footnotetext{
5 El número de retardos se seleccionó utilizando el criterio de información de Akaike (AIC).

6 Véanse en el cuadro A2.1 del anexo A2 las pruebas de Dickey-Fuller aumentadas para las variables y residuales. Se llevó a cabo esta prueba en el caso de las residuales para comprobar su relación de cointegración. Se informa sobre el valor crítico correcto para cada prueba.
} 
Utilizando un número diferente de retardos, como indican las pruebas de cociente de verosimilitudes (likelihood ratio) (un retardo), el criterio de información bayesiano de Schwarz (un retardo), el criterio de información de Akaike (tres retardos), el error de predicción final (tres retardos) y el criterio de información de Hannan-Quinn (dos retardos), no podemos rechazar la hipótesis nula de la no cointegración. Esta solo se ve rechazada cuando seleccionamos cero retardos, pero ningún criterio de selección de retardos sugiere hacerlo. A continuación, realizamos un análisis de vectores autorregresivos (VAR) de las diferencias entre estas variables. Puesto que los países analizados son grandes productores de metales, las tendencias de conflicto comunes a todos los países también pueden causar alguna variación en los precios, debido a una producción mayor o menor. Se estimó un modelo VAR, utilizando un retardo (como parecen indicar todas las pruebas llevadas a cabo) sin imponer ninguna restricción. El modelo VAR(1) utilizado fue el siguiente:

$$
\begin{gathered}
y_{1, t}=c_{1}+a_{1,1} y_{1, t-1}+a_{1,2} y_{2, t-1}+e_{1, t} \\
y_{2, t}=c_{1}+a_{2,1} y_{1, t-1}+a_{2,2} y_{2, t-1}+e_{1, t}
\end{gathered}
$$

donde $y_{1, t}$ es la primera diferencia del índice de precios de los metales básicos de la IFS e $y_{2, t}$ es la primera diferencia del primer factor del índice de noticias conflictivas estandarizado $\left(S C N_{i, t}\right)$ (denominado $F 1_{S C_{i, t}}$ en el cuadro A2.2). En el gráfico 5 se muestra la función de respuesta al impulso (véanse resultados adicionales en el cuadro A2.2 del anexo A2).

En el subgráfico 5C, se observa que una innovación estructural en los precios de los metales predice un incremento del índice de noticias conflictivas estandarizado en el siguiente trimestre. Esto no se estima con precisión, y si se realiza una descomposición de Cholesky (ordenando el índice de precios de los metales básicos primero), el coeficiente no difiere de 0 a niveles normales significativos, pero mantiene el signo. Se trata de una predicción interesante hecha a partir de un modelo VAR simple y podría ser una línea de investigación futura fructífera. También es congruente con los resultados de Dube y Vargas (2013) sobre las crisis de los precios del petróleo en Colombia.

\section{Gráfico 5}

Australia, Canadá, Chile y Perú: función de respuesta al impulso en los precios de los metales básicos y carga factorial principal

A. Respuesta al impulso en el índice de noticias conflictivas estandarizado frente al propio impulso en las noticias conflictivas (índice estandarizado)

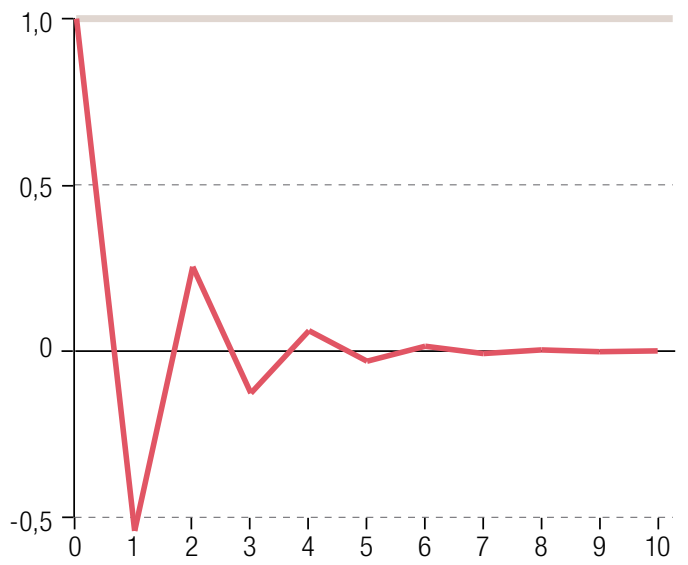

B. Respuesta al impulso del índice de precios de los metales básicos frente al índice de noticias conflictivas estandarizado

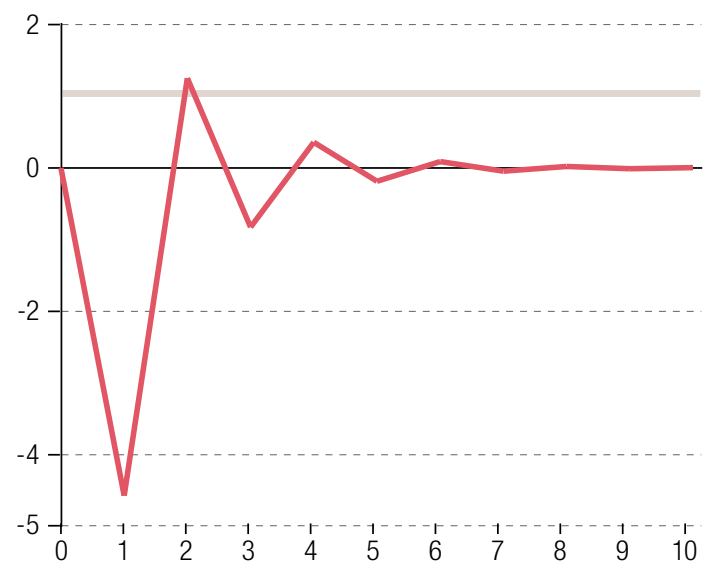


Gráfico 5 (conclusión)

C. Respuesta al impulso del índice de noticias conflictivas estandarizado frente al índice de precios de los metales

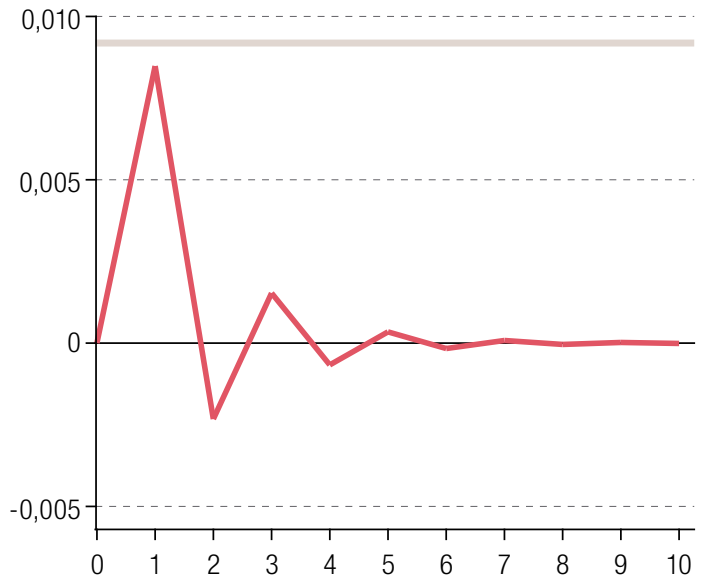

D. Respuesta al impulso en el índice de precios de los metales frente al propio impulso en el precio de los metales

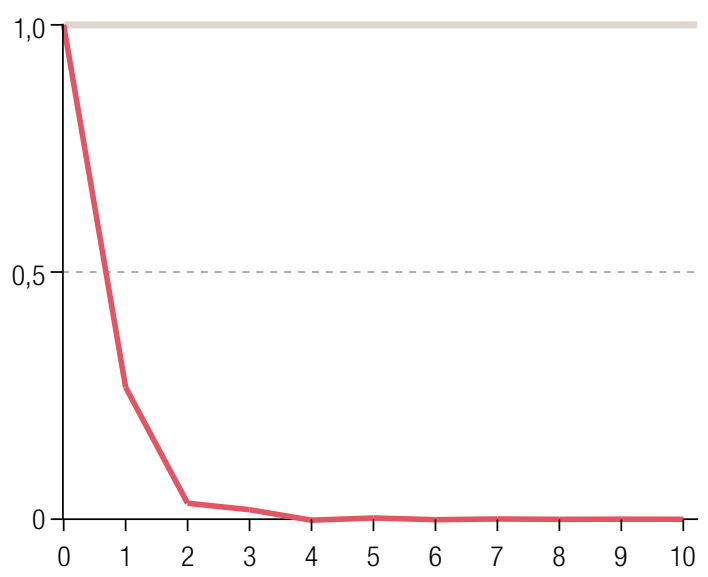

Fuente: Elaboración propia.

Nota: Gráficos por función de respuesta al impulso, variable de impulso y variable de respuesta.

\section{Regresiones de datos de panel}

En esta sección se incluye un análisis en profundidad de la relación entre los conflictos, los precios, las rentas procedentes de la minería y el desempeño económico general, a partir de nuestra base de datos de panel y la correlación de nuestros índices con los precios de los metales básicos, las rentas procedentes de la minería como porcentaje del PBI y el PBI per cápita (en dólares a valores constantes de 2010). Estas dos últimas variables se extrajeron de la base de datos Indicadores del Desarrollo Mundial (Banco Mundial, 2019). Se utilizan datos anuales debido a que el patrón temporal de nuestros principales determinantes potenciales también es anual.

El análisis se realiza sobre los índices de noticias conflictivas estandarizado $\left(S C N_{i, t}\right)$, de intensidad del conflicto $\left(C_{i, t}\right)$, de noticias violentas como porcentaje de las noticias conflictivas y de intensidad del conflicto violento $\left(V C_{i, t}\right)$. Todos los modelos de regresión utilizan estimadores de efectos fijos de país y $a \tilde{n} 0^{7}$. Nuestro modelo de regresión preferido es el siguiente:

$$
\ln \left(S C N_{i, t}\right)=\beta_{0}+\beta_{1} \ln \left(M R_{i, t}\right)+\beta_{2} \ln \left(G D P_{i, t}\right)+f_{i}+f_{t}+\epsilon_{j, t}
$$

donde $M R_{i, t}$ son las rentas procedentes de la minería como porcentaje del PBI del país $i$ en el año $t$; $G D P_{i, t}$ es el PBI de cada país para cada año, y $f_{i}$ y $f_{t}$ son los efectos fijos de tiempo y país. Sin embargo, también probamos a reemplazar con el índice de precios de los metales básicos y el nivel del PBI con la tasa de crecimiento del PBI. Todas las regresiones deben interpretarse con cautela, pues en nuestro análisis no hemos tenido en cuenta adecuadamente la causalidad inversa. Sin embargo, el tipo de conflicto no parece ser un factor tan importante para causar una caída de la producción que afecte a los precios de los productos básicos. Los resultados principales se presentan en el cuadro 5.

\footnotetext{
7 Una inspección visual del índice de noticias conflictivas estandarizado $\left(S C N_{i, t}\right)$ podría indicar que esas series no son I(1). No obstante, aplicamos la prueba de Im, Pesaran y Shin en Stata utilizando un retardo seleccionado mediante el AlC, lo que nos llevó a rechazar, con un valor $p$ de 0,0185, la hipótesis nula de que todos los paneles contienen una raíz unitaria.
} 
Cuadro 5

Regresiones de efectos fijos por país y año del índice de noticias conflictivas estandarizado

\begin{tabular}{|c|c|c|c|c|c|c|}
\hline Variables & $\begin{array}{c}1 \\
\text { In } \\
\text { (indice de noticias } \\
\text { conflictivas } \\
\text { estandarizado (scn)) }\end{array}$ & $\begin{array}{c}2 \\
\text { In } \\
\text { (indice_scn) }\end{array}$ & $\begin{array}{c}3 \\
\text { In } \\
\text { (indice_scn) }\end{array}$ & $\begin{array}{c}4 \\
\text { In } \\
\text { (indice_scn) }\end{array}$ & $\begin{array}{c}5 \\
\text { In } \\
\text { (indice_scn) }\end{array}$ & $\begin{array}{c}6 \\
\text { In } \\
\text { (indice_scn) }\end{array}$ \\
\hline \multirow{2}{*}{$\begin{array}{l}\text { In } \\
\text { (rentas } \\
\text { procedentes de } \\
\text { la minería como } \\
\text { porcentaje del PBI) }\end{array}$} & $0,222^{\star \star \star}$ & $0,214^{\star \star \star}$ & $0,226^{\star \star \star}$ & & & \\
\hline & $(0,0709)$ & $(0,0725)$ & $(0,0751)$ & & & \\
\hline \multirow{2}{*}{$\begin{array}{l}\text { In } \\
\text { (indice de precios } \\
\text { de los metales) }\end{array}$} & & & & 0,0559 & $-0,00492$ & 0,0449 \\
\hline & & & & $(0,0789)$ & $(0,0972)$ & $(0,0814)$ \\
\hline \multirow{2}{*}{$\begin{array}{l}\text { In } \\
\text { (PBI per cápita en } \\
\text { dólares a valores } \\
\text { constantes } \\
\text { de 2010) }\end{array}$} & & 0,212 & & & 0,368 & \\
\hline & & $(0,317)$ & & & $(0,346)$ & \\
\hline \multirow{2}{*}{$\begin{array}{l}\text { Primera } \\
\text { diferencia_In } \\
\text { (PBI per cápita en } \\
\text { dólares a valores } \\
\text { constantes } \\
\text { de 2010) } \\
\end{array}$} & & & $-0,385$ & & & 1,224 \\
\hline & & & $(1,844)$ & & & $(1,958)$ \\
\hline \multirow[t]{2}{*}{ Constante } & $-0,372^{\star \star \star}$ & $-2,433$ & $-0,364^{\star \star *}$ & $-0,620$ & $-3,946$ & $-0,600$ \\
\hline & $(0,0668)$ & $(3,085)$ & $(0,0785)$ & $(0,380)$ & $(3,146)$ & $(0,385)$ \\
\hline Observaciones & 52 & 52 & 52 & 52 & 52 & 52 \\
\hline R cuadrado & 0,412 & 0,420 & 0,413 & 0,248 & 0,272 & 0,256 \\
\hline $\begin{array}{l}\text { Efectos por país } \\
\text { y año }\end{array}$ & Sí & Sí & Sí & Sí & Sí & Sí \\
\hline Número de países & 4 & 4 & 4 & 4 & 4 & 4 \\
\hline
\end{tabular}

Fuente: Elaboración propia.

Nota: Significación estadística del coeficiente: ${ }^{* \star \star} p<0,01,{ }^{\star \star} p<0,05,{ }^{\star} p<0,1$. Los errores estándares robustos se muestran entre paréntesis.

Hallamos una covarianza positiva y significativa entre las rentas procedentes de la minería como porcentaje del PBI y el índice de noticias conflictivas estandarizado. Esa variable es estable y mantiene su signo y su significación aun al incluir el PBI per cápita o la primera diferencia del PBI per cápita. Las rentas procedentes de la minería como porcentaje del PBI podrían ser más precisas, dado que varían por país, mientras que el índice de precios de los metales básicos es el mismo para todos los países analizados.

Una posible interpretación de nuestros resultados es que un nivel elevado de rentas procedentes de la minería provoca más conflictos, porque diferentes agentes intentan apropiarse de un mayor porcentaje de dichas rentas. Estos agentes pueden incluir al gobierno, las empresas nacionales, las empresas extranjeras o los trabajadores. Esto concuerda con las conclusiones de los autores que han tratado la maldición de los recursos naturales mencionada anteriormente, como Brunnschweiler y Bulte (2009), así como con los resultados de Dube y Vargas (2013) en lo que respecta al efecto de rapacidad. Palazzo (2017) también llega a la misma conclusión en su artículo sobre bienes agropecuarios en la Argentina.

El análisis del índice de artículos de prensa violentos como razón del total de artículos de prensa sobre conflictos no revela ninguna relación clara entre esta variable y el índice de precios de los metales básicos, las rentas procedentes de la minería como porcentaje del PBI y el PBI per cápita (véase el cuadro A2.3). Además, nuestros intentos de hallar determinantes de la intensidad del 
conflicto y la intensidad del conflicto violento no tuvieron éxito. Por último, aplicamos el mismo modelo de regresión, añadiendo y cambiando algunas variables independientes, pero ninguna de ellas resultó estadísticamente significativa ${ }^{8}$.

Todos estos resultados deben interpretarse con cautela, dado que trabajamos con una muestra pequeña y no abordamos apropiadamente los problemas de endogeneidad. Sin embargo, la correlación entre el índice de noticias conflictivas estandarizado y las rentas procedentes de la minería resulta interesante, pues coincide con los resultados de los trabajos que analizan la maldición de los recursos. Estos resultados refuerzan nuestros hallazgos anteriores: el aumento de los precios de los metales básicos puede predecir el factor común del índice de noticias conflictivas estandarizado $\left(S_{C N} N_{i, t}\right)$.

\section{Conclusiones}

En este artículo, se examinan la incidencia y la intensidad de los conflictos sociales relacionados con actividades extractivas en cuatro países mineros, aplicando técnicas de minería de textos a artículos publicados en los principales periódicos de cada país durante el período 2003-2016. El resultado más importante de este análisis son los índices elaborados para medir el conflicto y la intensidad del conflicto, que se encuentran disponibles para futuras investigaciones.

Debe tenerse en cuenta que las diferencias que encontramos entre los países pueden haber surgido debido, entre otras cosas, a sesgos en los medios de comunicación, a las distintas maneras de expresarse y comunicar las noticias en función del idioma utilizado, y a preferencias o intereses dispares de los lectores de los diferentes periódicos, que pueden influir en la manera en que los periodistas redactan sus artículos. Detectar estos sesgos es el primer paso para tratar de comprender y medir los conflictos civiles relacionados con actividades extractivas. A pesar de estos sesgos potenciales, a continuación resumimos los principales resultados de este estudio.

En primer lugar, encontramos diferencias entre Australia, el Canadá, Chile y el Perú, así como entre los distintos años analizados en el caso de cada país. En los países desarrollados, el nivel de conflictivas es estable y siempre más elevado que el registrado en los países en desarrollo. En estos, la cobertura mediática de las actividades extractivas tiende a ser más favorable, si bien se detectaron algunos conflictos durante el período considerado.

En segundo lugar, calculamos las diferencias entre ambos grupos de países. Con respecto a los países desarrollados, el nivel de noticias conflictivas en el Canadá siguió una trayectoria ascendente (aunque empezaba en un nivel más bajo) y la intensidad del conflicto resultó más elevada que en Australia. En cuanto a los países en desarrollo, tanto el nivel como la intensidad del conflicto social se incrementaron en el Perú, mientras que en Chile no se detectó una tendencia clara, si bien cabe subrayar que la intensidad del conflicto se mantuvo baja y estable. Al categorizar los conflictos sociales como violentos o no violentos, hallamos un sesgo en los países en desarrollo hacia los conflictos violentos.

En tercer lugar, el análisis de los conflictos sociales relacionados con las actividades extractivas arrojó algunos resultados interesantes cuando se desagregó por áreas administrativas subnacionales. Descubrimos que la cantidad de artículos de prensa obtenidos a través de la técnica de la minería de textos estaba correlacionada con la importancia económica de las industrias extractivas en un área determinada. Por otra parte, el nivel medio de conflictos en los países desarrollados en la totalidad del período analizado fue bastante homogéneo en las distintas áreas administrativas, mientras que

\footnotetext{
8 Por ejemplo, hicimos una prueba para averiguar si el ciclo político afecta los conflictos civiles relacionados con las actividades extractivas. La variable del ciclo político se creó definiendo una variable indirecta igual a 1 si se habían celebrado elecciones generales en un país en un año determinado. Si las elecciones se habían llevado a cabo antes del primer trimestre, el año anterior se definía como año electoral. Los resultados están disponibles a solicitud de los interesados.
} 
las disparidades en los países en desarrollo fueron profundas. La intensidad del conflicto también resultó bastante homogénea en los países desarrollados y heterogénea en los países en desarrollo, especialmente en el Perú.

En cuarto lugar, exploramos en qué medida se interrelacionaban los conflictos nacionales. Se llevó a cabo un análisis de factor común que indicó que los niveles de conflicto venían determinados por la situación particular de cada país. Sin embargo, un factor común positivo está relacionado con el índice general de precios de los metales básicos, por lo que un aumento de los precios de los metales podría predecir el incremento del nivel de conflictos.

Por último, se comprobó este resultado por medio de regresiones de datos de panel. Se encontró una relación positiva entre el índice de noticias conflictivas estandarizado y las rentas procedentes de la minería como porcentaje del PBI. Este resultado está en consonancia con el efecto de rapacidad identificado por Dube y Vargas (2013) y los trabajos que analizan la maldición de los recursos naturales. No obstante, estas conclusiones deben interpretarse con cautela, dado que nuestra muestra es reducida y no abordamos de manera adecuada los problemas de endogeneidad.

\section{Bibliografía}

Arezki, R. y F. van der Ploeg (2007), "Can the natural resource curse be turned into a blessing? The role of trade policies and institutions", IMF Working Paper, N 07/55, Washington, D.C., Fondo Monetario Internacional (FMI).

Aromí, J. (2013), "Media news and the stock market: evidence for Argentina" [en línea] https://docplayer. net/56643583-Media-news-and-the-stock-market-evidence-for-argentina.html.

Baker, S., N. Bloom y S. Davis (2013), "Measuring economic policy uncertainty", Chicago Booth Research Paper, No 13-02, enero.

Banco Mundial (2019), "Indicadores del Desarrollo Mundial" [base de datos en línea] https://databank. bancomundial.org/reports.aspx?source=world-development-indicators.

(2011), The Changing Wealth of Nations: Measuring Sustainable Development in the New Millennium, Washington, D.C.

(2009), ¿Dónde está la riqueza de las naciones? Medir el capital para el siglo XXI, Washington, D.C.

Brunnschweiler, C. y E. Bulte (2009), "Natural resources and violent conflict: resource abundance, dependence, and the onset of civil wars", Oxford Economic Papers, vol. 61, № 4, octubre.

Collier, P. y A. Hoeffler (2005), "Resource rents, governance, and conflict", The Journal of Conflict Resolution, vol. 49, N 4, agosto.

(2000), "Greed and grievance in civil war", Policy Research Working Paper, № 2355, Washington, D.C., Banco Mundial.

(1998), "On economic causes of civil war", Oxford Economic Papers, № 50.

Dow Jones (2020), "Factiva" [en línea] https://professional.dowjones.com/factiva/.

Dube, O. y J. Vargas (2013), "Commodity price shocks and civil conflict: evidence from Colombia", The Review of Economic Studies, vol. 80, $\mathrm{N}^{\circ} 4$, octubre.

Evans, A. (2010), "Resource scarcity, climate change and the risk of violent conflict", World Development Report 2011 Background Papers, № 62050, Washington, D.C., Banco Mundial, septiembre.

García, D. (2013), "Sentiment during recessions", The Journal of Finance, vol. 68, № 3, junio.

Giordano, M., M. Giordano y A. Wolf (2005), "International resource conflict and mitigation", Journal of Peace Research, vol. 42, № 1, enero.

Gupta, V. y G. Lehal (2009), "A survey of text mining techniques and applications", Journal of Emerging Technologies in Web Intelligence, vol. 1, No 1, agosto.

Haber, S. y V. Menaldo (2011), "Do natural resources fuel authoritarianism? A reappraisal of the resource curse", American Political Science Review, vol. 105, № 1, febrero.

Hirschman, A. (1977), "Enfoque generalizado del desarrollo por medio de enlaces, con referencia especial a los productos básicos", El Trimestre Económico, vol. 44, № 173.

Humphreys, M. (2005), "Natural resources, conflict, and conflict resolution: uncovering the mechanisms", 
The Journal of Conflict Resolution, vol. 49, № 4, agosto.

Leite C. y J. Weidmann (1999), "Does mother nature corrupt? Natural resources, corruption, and economic growth", IMF Working Paper, № 99/85, Washington, D.C., Fondo Monetario Internacional (FMI), julio.

Mehlum, H., K. Moene y R. Torvik (2006), "Cursed by resources or institutions?", The World Economy, vol. 29, $\mathrm{N}^{\circ} 8$, agosto.

Naciones Unidas (2014), System of Environmental-Economic Accounting 2012: Experimental Ecosystem Accounting (ST/ESA/STAT/Ser.F/112), Nueva York. (s/f), "System of Environmental-Economic Accounting" [en línea] https://seea.un.org/es.

Nafziger, E. y J. Auvinen (2002), "Economic development, inequality, war, and state violence", World Development, vol. 30, № 2, febrero.

Palazzo, G. (2017), "Minería de datos, conflictos sociales y explotación de recursos naturales: un aporte metodológico", Compendium: Cuadernos de Economía y Administración, vol. 4, № 7. (s/f), "Original databases: conflicts and natural resources" [base de datos en línea] https://sites.google. com/view/gabrielmpalazzo/original-databases.

PNUD (Programa de las Naciones Unidas para el Desarrollo)/Fundación UNIR (2012), "La protesta social en América Latina", Cuaderno de Prospectiva Política, № 1, Buenos Aires, Siglo XXI.

Prebisch, R. (1949), El desarrollo económico de la América Latina y sus principales problemas (E/CN.12/89), Santiago, Comisión Económica para América Latina y el Caribe (CEPAL).

Robinson, J., R. Torvik y T. Verdier (2006), "Political foundations of the resource curse", Journal of Development Economics, vol. 79, № 2.

Sachs, J. y A. Warner (1995), "Natural resource abundance and economic growth", NBER Working Paper, № 5398, Cambridge, Oficina Nacional de Investigaciones Económicas (NBER), diciembre.

Singer, H. (1950), "Comercio e inversión en países poco desarrollados: distribución de las ganancias entre los países inversores y los deudores", El Trimestre Económico, vol. 17, Nㅜ 66.

Sinnott, E., J. Nash y A. de la Torre (2010), Los recursos naturales en América Latina y el Caribe: ¿más allá de bonanzas y crisis?, Washington, D.C., Banco Mundial.

Tetlock, P. (2007), "Giving content to investor sentiment: the role of media in the stock market", The Journal of Finance, vol. 62, $\mathrm{N}^{\circ} 3$, junio. 


\section{Anexo A1}

\section{Palabras hostiles y violentas utilizadas para el ejercicio de minería de textos}

Palabras hostiles en inglés: abhor, abolish, abrasive, abscond, absentee, abuse, accost, accursed, accusation, accuse, acrimonious, acrimony, admonish, adversary, afflict, aggravate, aggravation, aggression, aggressive, aggressiveness, aggressor, aggrieve, agitator, alienate, allegation, altercation, ambush, anarchist, anarchy, anger, angry, anguish, animosity, annihilate, annihilation, annoy, annoyance, antagonism, antagonist, antagonistic, antagonize, argue, argument, arm, armed, arrest, arrow, assail, assailant, assassin, assassinate, assault, attack, attacker, austere, avenge, aversion, avert, avoid, avoidance, bandit, banish, barbarian, barbarous, bastard, battle, battlefield, beastly, beat, behead, belie, belittle, belligerent, belt, bereave, berserk, besiege, betray, betrayal, beware, bit, bitchy, bite, bitter, blame, blind, block, bloodshed, bloodthirsty, blurt, bomb, bombard, bombardment, bother, bout, boycott, brandish, brawl, breach, break, bristle, broke, brusque, brutality, brute, brutish, bug, bullet, burglar, burglary, butchery, callous, cannibal, cannon, capture, cataclysm, caught, censure, challenge, charge, chase, chastise, cheat, chide, chip, choke, clash, collide, collision, combat, combatant, compel, compete, competition, competitive, competitor, complain, complaint, compulsion, conceal, conceit, condemn, condemnation, condescending, condescension, conflict, confront, confrontation, conspiracy, constrain, contaminate, contamination, contempt, contemptible, contemptuous, contend, contest, contradict, convict, corruption, coup, cranky, crass, criminal, cripple, critic, criticize, crooked, crop, cross, cruel, cruelty, crush, crushing, curse, curt, cut, cynical, dagger, damage, damn, damnable, damned, deadlock, deadly, deceit, deceitful, deceive, deception, deceptive, defeat, defensive, defiance, defiant, defile, defy, degrade, demean, demolish, demon, demoralize, denial, denounce, deny, deplore, depose, deprive, deride, derision, derogatory, desert, despise, destroy, destruction, destructive, deterrent, detest, devastate, devastation, devil, devilish, devious, diabolic, diabolical, disagree, disagreeable, disagreement, disapprove, disavow, disavowal, disbelief, discord, discordant, discourage, discredit, discrepant, discrimination, disgust, dislike, dismiss, disobedience, disobedient, displeasure, dispose, disputable, dispute, disrupt, disruption, dissatisfied, dissent, dissention, distort, distrust, disturb, disturbance, ditch, divorce, drag, dump, egotistical, endanger, enemy, enforce, engulf, enrage, entanglement, epithet, escape, exception, exclude, exclusion, excommunication, execute, execution, exile, expel, exploit, fail, fallout, ferocious, ferocity, feud, fiend, fierce, fight, fighter, fire, fist, fled, floor, foe, fool, force, foreboding, fought, frighten, frown, frustrate, fun, furious, fury, germ, goddamn, grab, grapple, grenade, grudge, grumble, guerrilla, gun, gunmen, hamper, hang, harass, harassment, harm, harsh, hassle, hate, hater, hatred, haunt, heartless, hedge, heinous, hidden, hide, hinder, hindrance, hit, horrify, horror, hostile, hostility, humiliate, hunt, hunter, hurt, hustle, hustler, impair, impatience, impede, impediment, implicate, indictment, indignation, infect, infection, infiltration, inflame, infringement, infuriate, inhibit, inhibition, inhumane, injunction, injurious, injury, insolence, insolent, interfere, interference, interrupt, interruption, intrusion, irk, irritable, irritation, jagged, jail, jeer, jeopardize, jerk, kick, kidnap, kill, killer, knife, knock, laid, lawless, liar, lie, limit, liquidate, liquidation, litigant, litigation, lying, mad, malice, malicious, malignant, mangle, manslaughter, marksman, massacre, merciless, misbehave, mislead, missile, mob, molest, monster, monstrous, murder, murderous, mutter, nag, nasty, naughty, needle, negate, neglect, nigger, nightmare, obliterate, obnoxious, obstinate, obstruct, offend, offender, offensive, ominous, opponent, oppose, opposition, oppress, oppression, ostracize, oust, outlaw, outrage, pan, parasite, pass, penalty, penetrate, penetration, persecute, persecution, pinch, pistol, plague, plight, poisonous, pollute, posse, prejudice, pretend, pretence, prosecution, protest, provocation, provoke, prowl, punch, punish, push, quarrel, quarrelsome, quibble, rage, raid, raise, ravage, reactive, rebel, rebellion, rebellious, rebuff, rebuke, rebut, recalcitrant, refusal, refuse, reject, rejection, renounce, renunciation, repel, reproach, 
repulse, resent, resentful, resentment, resist, resistance, restrain, restrict, retaliate, retard, revenge, revolt, revolution, revolutionary, rid, ridicule, rifle, rip, rival, rivalry, robber, robbery, rogue, ruffian, ruinous, rumple, rupture, ruthless, ruthlessness, sabotage, sarcasm, sarcastic, savage, scandalous, scare, scared, scold, scorch, scorn, scornful, scoundrel, scowl, scuffle, seethe, segregation, sever, shadow, shaft, shatter, shock, shoot, shot, shove, shred, shrew, shrug, shudder, shun, shut, sick, siege, sinister, skirmish, slam, slander, slanderer, slanderous, slap, slash, slaughter, slayer, sleazy, slight, sly, smack, smash, smear, snarl, snatch, spank, spear, spite, spiteful, split, spoil, stab, stall, stamp, startle, steal, stern, stifle, sting, stole, stolen, stone, stop, storm, stormy, strangle, strife, strike, stringent, strip, struck, struggle, stubborn, stubbornly, stubbornness, stun, subdue, subversion, subvert, sunder, suppress, suppression, suspect, suspicion, suspicious, sword, taboo, taint, tamper, tantrum, taunt, tear, tease, temper, tempest, tense, terrorism, terrorize, theft, thief, thorny, thrash, threat, threaten, thwart, tire, tired, TNT, torment, tough, traitor, trample, trap, treacherous, treachery, treason, treasonous, trick, trigger, turbulent, ultimatum, undermine, unfair, unjust, unjustified, unleash, unruly, unsafe, untruth, unwilling, unwillingness, uprising, upset, usurp, vengeance, venom, venomous, vicious, victim, vie, villain, violate, violation, violence, violent, wait, walk, war, warlike, warrior, weapon, weed, wench, whack, whine, whip, wicked, wickedness, wily, witch, witchcraft, withheld, withhold, withstand, worry, wound, wrath, wreck, wrestle, wrong.

Palabras violentas en inglés: aggressive, aggressiveness, ambush, arm, armed, assault, attack, beat, belt, bloodthirsty, bomb, brutality, bullet, butchery, crush, crushing, deadly, fire, guerrilla, gun, gunmen, kill, killer, monster, monstrous, murder, rebel, rebellion, slash, slayer, violence, violent, warrior, whip.

Palabras hostiles en español: abatir, abdicación, abdicar, abofetear, abolición, abolir, abominación, abominado, abominar, aborrecer, aborrecido, aborrecimiento, aborrezco, abrumar, abuchear, abucheo, abusar, abusivo, abuso, abyecto, acéfalo, acérrimamente, acérrimo, aciago, acobardar, acogotar, acorralamiento, acorralar, acosamiento, acribillar, acritud, acuchillar, acusación, adulterino, aflicción, afligido, afligir, agitación, agitador, agobiar, agobio, agolpamiento, agraviante, agraviar, agravio, agredir, agresión, agresividad, agresivo, agresor, ahogamiento, ahogar, ahogo, ahorcamiento, ahorcar, ahuyentar, ajusticiar, ajusticiamiento, alabarda, alboroto, alegación, alegato, alevosía, alienar, altercado, alzamiento, alzarse, embaucador, amedrentar, amenaza, amenazador, amenazar, ametralladora, ametrallar, amilanar, amohinado, amonestar, amotinador, amotinarse, amputar, anarquía, anárquico, anarquista, anatema, angustia, angustiado, animadversión, animosidad, aniquilación, aniquilamiento, aniquilar, anormalidad, antagónica, antagonismo, antagonista, antihigiénico, anticristo, apedrear, apercibir, apesadumbrado, apesadumbrar, aporrear, aprehender, apremio, apresamiento, apresar, aprieto, aprisionamiento, aprisionar, apuntar, apuñalar, arma, armamento, armas, arpía, arrebatar, arredrar, arremeter, arremetida, arrestar, arresto, arrinconar, arrogancia, arrogante, artero, artimaña, asaltado, asaltador, asaltante, asaltar, asalto, asco, asechada, asechanza, asechar, asediar, asesinar, asesinato, asesino, asfixia, asfixiar, asolación, asolar, asustar, atacante, atacar, ataque, atemorizar, atentado, atentar, aterrador, aterrar, aterrarse, aterrorizar, atizo, atormentado, atormentar, atosigamiento, atosigar, atracador, atracando, atracar, atraco, atraco, atrocidad, atroz, austero, avasallamiento, avasallar, bandolero, bastardo, batalla, batallador, batallando, batallar, bayoneta, belcebú, bélico, belicosidad, belicoso, beligerante, bellaco, bestialidad, blasfemar, blasfemia, boicot, boicotear, boicoteo, bomba, bombardear, bombardeo, bravucón, bribón, bronca, cacheteada, cachetear, cachiporra, calamidad, calumnia, calumniador, calumniando, calumniar, calumnioso, calvario, camorrista, canalla, carabina, castigar, castigo, cataclismo, catástrofe, caustico, celada, censura, censurar, cercenar, chantaje, chantajista, chiflado, chiflarse, cicatero, cínica, claudicar, cleptómano, coacción, coercer, cohecho, cohibir, cólera, colérico, coletazo, combate, combatiendo, combatiente, combatir, combatividad, combativo, complot, complotar, compulsión, condena, condenado, condenar, confabulación, confabular, confiscación, confiscar, conflagración, conflagración, conflicto, confrontación, confrontar, conjuración, conjurar, conminación, 
conspiración, conspirador, conspirar, constreñir, consternar, constreñir, contaminación, contaminado, contaminar, contender, contendiente, contestatario, contienda, contradecir, contradictorio, contraponer, contraproducente, contrincante, controversia, controvertir, contumacia, convicto, convulsión, convulsionar, corrompido, corrupción, corruptela, crimen, criminal, crispar, cruel, crueldad, cuartelazo, cuatrero, cuchillo, cuestionar, culpa, culpable, culpar, daga, damnificación, damnificado, damnificar, dañino, decapitar, defensivo, defraudado, defraudando, defraudar, defraudo, degollar, degollina, degradar, degradarse, dejadez, delincuente, demonio, denegar, denigración, denigrante, denigrar, denigrativo, denuncia, denunciar, deponer, deportación, deportar, depravado, depredar, derogación, derribar, derrotar, derruir, desacato, desacreditar, desacuerdo, desafiante, desafiar, desafuero, desagradable, desagraviar, desalentar, desalmado, desalojar, desalojo, desanimar, desaparecer, desapoderamiento, desaprobación, desaprobar, desaprueba, desasosiego, desastre, desatender, desatinar, desautorización, desautorizar, desavenencia, desazón, desbaratar, descabezado, descalabrar, descalificación, descalificar, descarado, descaro, descontento, descorazonar, descuartizar, descuidar, descuidero, descuido, desdecir, desdén, desdeñoso, desdichado, desencantado, desengañar, desequilibrarse, deserción, desertar, desertor, desesperación, desesperanzar, desestimar, desfalco, desfigurar, desgana, desgarrar, desgraciado, deshacer, deshacerse, deshecho, deshilachar, deshizo, deshonesto, deshonra, deshonrar, desidia, desigualdad, desilusión, desilusionar, desistimiento, desistir, desleal, deslealtad, desmantelado, desmantelar, desmembrar, desmentir, desmoralizar, desnucar, desobediencia, desobediente, desolación, desolar, desparecer, despecho, despectivo, despedazado, despedazar, despedido, despedir, despiadado, despidos, despojado, despojar, desposeer, déspota, despotismo, despotricar, despreciable, despreciado, despreciar, desprecio, desprestigiar, desquiciado, desquiciar, desquitar, desquitarse, desquite, destacamento, desterrado, desterrar, destierro, destituido, destituir, destripar, destrozado, destrozar, destrucción, destructivo, destructor, destruir, desunión, desunir, desvergonzado, desvergüenza, detención, detestable, detestar, detesto, detonación, detonador, detonar, detractar, desunión, devastación, devastar, diablo, diabólico, dictadura, difamación, difamador, difamar, difamatorio, difunto, dimisión, dimitir, díscolo, disconformidad, discordante, discordia, discrepancia, discrepante, discrepar, discriminación, discriminar, discusión, discutiendo, discutir, disensión, disentimiento, disentir, disgustado, disgustar, disgusto, disimulo, dislocación, dislocar, disolución, disoluto, disonancia, disputa, disputable, disputar, disputarse, distorsionar, disturbio, disuasivo, disuasorio, doblegarse, dolorido, dominación, embarullar, embate, embaucar, embestida, embestir, emboscada, emboscar, embrollar, embuste, embustero, embustir, emponzoñar, enajenar, enardecer, enardecido, encarcelamiento, encarcelar, encizañar, encolerizado, encolerizar, encrespar, enemigo, enemistad, enemistar, enemistarse, enervar, enfadado, enfadar, enfadarse, enfado, enfrentado, enfrentamiento, enfrentar, enfurecer, enfurecido, enfurecimiento, engañando, engañar, engaño, engañosa, engañoso, engatusar, engendro, engorro, engreimiento, enjuiciamiento, enjuiciar, enloquecer, enloquecido, enmascarar, enojado, enojar, enojo, ensañamiento, entorpecer, entristecer, envenenar, enviciar, envilecer, epidemia, escandalo, escaramuza, escarmentar, escarmiento, escarnio, escepticismo, escéptico, esclavitud, esclavizar, esclavo, escopeta, esfumarse, espada, espantar, espantarse, espinoso, espurio, estacazo, estafa, estafador, estafando, estafar, estafo, estallar, estallido, estigma, estigmatizar, estrago, estrangulación, estrangulamiento, estrangular, estremecer, estremecimiento, estremezco, estropeado, estropear, estuprar, evadir, evadirse, evasión, exabrupto, exasperación, exasperar, exclusión, exilio, expatriación, expatriado, expiación, explosión, explosionar, explosivo, explotar, expropiación, expulsar, expulsión, exterminar, exterminio, extinción, extirpar, extorsión, extralimitación, falsificación, falsificando, falsificar, fanfarrón, farfullar, farsa, farsante, fascismo, fastidiar, fastidio, fastidioso, fatal, fatídico, felonía, flagelar, flagelo, flecha, follón, forajido, forzado, forzar, fraccionar, fractura, fracturado, fracturar, fragmentar, francotirador, fratricida, fratricidio, fraude, frustración, frustrar, frustrarse, fuga, fugar, fugarse, funesto, furia, furioso, furtivo, fusil, fusilar, fusta, fusta, fustigar, gánster, garrocha, garrote, gatillo, golpe, golpeado, golpear, golpeo, golpismo, golpiza, granada, granuja, gresca, grilletes, grima, guerra, guerrear, guerrero, guerrilla, guerrillero, guillotinar, hastiado, hecatombe, herida, herido, herir, hipócrita, holocausto, homicida, homicidio, 
horrendo, horror, horrorizar, horrorizarse, hostigarían, hostigamiento, hostigar, hostil, hostilidad, hostilidades, hostilizar, huelga, huida, huir, humillante, humillar, hurtado, hurtando, hurtar, hurto, huyo, ignorado, ignorancia, ignorar, ilegal, ilegalidad, ilegitimo, ilícito, impedimento, impedir, impeler, impertinente, impiedad, implacable, implosión, impostor, improcedente, improperio, impúdico, impugnar, impurificar, imputación, imputar, inaceptable, incautación, accidentado, incinerar, incitación, incitar, incomodar, incomodidad, incompatibilidad, incompatible, inconmovible, inconsolable, incordiar, incordio, incredulidad, incrédulo, increpar, incriminación, incriminar, inculpación, inculpar, incumplimiento, indebido, indecente, indecoroso, indemnizar, indiferente, indignación, indignado, indigno, indisciplina, indocilidad, indolencia, indomable, ineptitud, inequidad, inescrupuloso, infamador, infamante, infamar, infame, infamia, infección, infectar, infecto, infestación, infestar, inficionar, ingrato, inhabilitación, inhabilitar, inhibición, inhibir, inhumano, injuria, injuriar, injurioso, injusticia, injustificado, injusto, inmerecido, inmolación, inmolado, inmolar, inmoral, inmundo, innoble, inoculación, inquina, insalubre, insano, insatisfecho, inseguro, insensible, insidia, insidioso, insolencia, insolente, instigación, instigador, instigar, insubordinación, insubordinado, insubordinarse, insultante, insultar, insulto, insumisión, insumiso, insurgente, insurrección, insurreccionarse, insurrecto, interferencia, interferir, intimidación, intimidar, intranquilidad, intranquilo, intransigencia, intransigente, intromisión, intrusión, inutilizar, invadido, invadir, invasión, iracundo, irascible, irracional, irracionalidad, irrazonable, irrespetuosidad, irreverencia, irrisorio, irritable, irritación, irritado, irritante, irritar, irrumpir, irrupción, jactancioso, jorobar, juzgar, laceración, lacerar, ladino, ladrón, lanceta, lapidar, lastimar, litigar, latigazos, látigo, leonino, letal, levantamiento, levantarse, levantisco, libertino, linchar, liquidación, liquidar, lisiar, litigante, litigar, litigio, llanto, lloriqueo, lucha, luchado, luchando, luchar, lucifer, luzbel, machacar, machete, madame, magnicida, magnicidio, magullado, magulladura, magullar, mal, malandrín, mal comportarse, maldad, maldecir, maldición, maldijo, maldito, maleante, maledicencia, malevolencia, malévolo, malhechor, malhumorado, malicia, malicioso, malignidad, maligno, malintencionado, malquerencia, malsano, maltratado, maltratar, maltrecho, malvado, malversación, malversado, malversando, malversar, malverso, marginar, masacrar, masacre, mascullar, matanza, matar, matón, mendacidad, mendaz, menoscabar, menospreciar, mentir, mentira, mentiroso, merodeo, metralleta, mezquino, milicia, militar, mintiendo, miserable, miseria, misil, mofa, mofar, molestar, molestia, molesto, molido, monstruo, monstruosa, monstruosidad, monstruoso, montaraz, mordacidad, mordaz, morder, mordida, mordió, mortal, mortandad, mortífero, mortificación, mortificado, mortificar, mosquetón, muerte, muertes, muerto, multa, munición, marginación, mutilar, navaja, nefasto, negligencia, nigromancia, nocivas, nocivo, obcecado, obligado, obligar, obliterar, obsceno, odiado, odiar, odio, ofender, ofendido, ofensa, ofensiva, ofuscación, ofuscado, ojeriza, ominoso, oponente, oponer, oponerse, oposición, opresión, oprimir, opuesto, ostentación, ostracismo, paliza, parricida, parricidio, patrulla, pécora, pegar, pego, pelea, peleón, pellizcar, pelotón, penado, penalizar, pendencia, pendenciero, penitencia, perjudicar, perjudicial, perjuicio, pernicioso, persecución, persecución, perseguir, persuadir, perturbación, perturbar, perversidad, perverso, pervertido, pervertir, pesadilla, pesadumbre, petulante, peyorativo, pillaje, pillar, piquete, pisotear, pistola, pistolero, pistolete, pleiteante, pleito, polémica, polemizar, polución, pólvora, ponzoña, ponzoñoso, porrazo, prejuicio, prescripción, presidiario, profanación, proscribir, proscripción, proscripto, protesta, protestar, provocador, proxeneta, pugna, pugnando, pugnar, pulverizar, punzar, puñal, puñalada, puñetazo, pútrido, quebrado, quebrantamiento, quebrantar, quebranto, quebrar, queja, quejar, quejarse, quejas, quejido, quejoso, querella, querellante, querellar, quiebra, quiebre, rabia, rabiar, rabieta, rapiñar, rapto, rastrero, ratero, reaccionario, rebelarse, rebelde, rebeldía, rebelión, recaída, recalcitrante, recelo, rechazar, rechazo, reclamar, reclamo, recluir, reclusión, recluso, recriminación, recriminar, refrenar, refriega, refunfuño, refutar, regañar, regaño, regicidio, rehuir, rehusar, rémora, rencor, rencoroso, renegar, renuncia, renunciar, repeler, reprobar, reprender, reprensión, represalia, represión, reprimenda, reprimir, reprobar, reprochar, reproche, repudiar, repudio, repugnancia, repugnante, repugnar, repulsión, repulsivo, resarcimiento, resarcir, resentido, resentimiento, resentir, resistencia, resistente, resistir, responsabilizar, resquemor, restringir, reticente, retorcido, retrogrado, revancha, reventado, reventar, revocación, revuelta, reyerta, rezongar, ridiculizar, ridículo, rifle, riñendo, rival, rivalidad, rivalizar, 
robado, robando, robar, robo, rufián, ruin, ruina, ruinoso, ruptura, sablista, sabotaje, sabotear, sacrificar, sacrificio, sádico, sadismo, salvajada, salvaje, sanguinario, saqueador, saqueo, satán, satanás, satánico, secuestrado, secuestrar, secuestro, sedición, sedicioso, segregación, segregar, sentencia, siniestro, sinvergüenza, soborno, socarronería, sofoco, sojuzgar, soliviantar, sollozo, someter, sopapear, soslayar, sospecha, sospechar, sospechoso, sublevación, sublevado, sublevarse, subversión, subversivo, subvertir, subyugar, sumisión, suspicacia, suspicaz, tabú, temerario, tergiversar, terrorismo, terrorista, tiranía, tiranizar, tirotear, tiroteo, tirria, torturado, torturar, tosigo, totalitarismo, toxico, tozudez, trágico, traición, traicionar, traicionero, traidor, traidores, tralla, trampa, trampear, tramposo, transgresión, transgresor, trastornado, trastornar, trastornarse, traumar, traumatismo, trifulca, triturar, trompazo, truhan, tumulto, turba, turbulento, ultimátum, ultrajar, ultraje, ultrajante, usurero, usurpado, usurpando, usurpar, usurpo, vandalismo, vapuleado, vapulear, vendetta, venganza, viciar, vicioso, victima, vil, vileza, vilipendio, villano, vindicta, violación, violar, violencia, violento, virulento, vividor, zaheridor, zaherir, zozobra, zurriago.

Palabras violentas en español: agresivo, combativo, provocador, violento, agresividad, belicosidad, combatividad, provocación, emboscada, celada, trampa, asechanza, artería, artimaña, emboscar, trampear, asechar, armas, armamento, armado, asaltar, atracar, robar, agredir, acometer, irrumpir, invadir, ataque, embate, irrupción, combate, lucha, agresión, golpear, golpe, sanguinario, choque, asalto, atropello, atentado, coletazo, bomba, explosivo, granada, munición, bala, brutalidad, bestialidad, ferocidad, crueldad, atrocidad, monstruosidad, irracionalidad, vandalismo, salvajada, grosería, masacre, matanza, mortandad, hecatombe, catástrofe, degollina, aplastar, triturar, reventar, destripar, moler, aplastamiento, mortal, mortífero, letal, fatídico, fatal, funesto, disparar, tirotear, ametrallar, despedir, expulsar, destituir, guerrilla, guerrillero, milicia, arma, pistola, revólver, pistolete, ametralladora, metralleta, pistolero, atracador, bandido, forajido, delincuente, gánster, terrorista, asesino, matar, asesinar, ahorcar, ahogar, decapitar, desnucar, degollar, fusilar, guillotinar, asfixiar, electrocutar, envenenar, linchar, asesinato, crimen, homicidio, delito, muerte, parricidio, fratricidio, magnicidio, regicidio, criminal, homicida, monstruo, engendro, deforme, monstruosa, rebelarse, incitar, sublevarse, insubordinarse, levantarse, alzarse, amotinarse, insurreccionarse, rebelión, levantamiento, revuelta, alzamiento, revolución, subversión, conspiración, conjuración, sedición, insurrección, motín, acuchillar, apuñalar, lesionar, violencia, exabrupto, coacción, profanación, furia, ensañamiento, violación, implacable, furioso, guerrero, soldado, militar, látigo, azote, fusta, tralla, vergajo, flagelo, zurriago, latigazos, azotando, litigar, azotar, fustigar, flagelar. 


\section{Anexo A2}

Cuadro A2.1

Prueba de raíz unitaria de Dickey-Fuller aumentada

\begin{tabular}{lcccc}
\hline Variable & Número de retardos & Te Student & Valor crítico 1\% & Valor crítico 5\% \\
\hline Índice de precios de los metales & retardos(2) & $-2,106$ & $-3,587$ & $-2,933$ \\
\hline D. Índice de precios de los metales (primera diferencia) & retardos(1) & $-4,379$ & $-3,587$ & $-2,933$ \\
\hline $\begin{array}{l}\text { Factor núm. 1: Índice de noticias conflictivas } \\
\text { estandarizado }\end{array}$ & retardos(3) & $-1,927$ & $-3,628$ & $-2,95$ \\
\hline $\begin{array}{l}\text { D. Factor núm. 1: Índice de noticias conflictivas } \\
\text { estandarizado (primera diferencia) }\end{array}$ & retardos(4) & $-4,407$ & $-3,655$ & $-2,961$ \\
\hline Error predicho & retardos(0) & $-4,613$ & $-4,124$ & $-3,461$ \\
\hline Error predicho & retardos(1) & $-2,707$ & $-4,124$ & $-3,461$ \\
\hline Error predicho & retardos(2) & $-1,534$ & $-4,124$ & $-3,461$ \\
\hline Error predicho & retardos(3) & $-1,231$ & $-4,124$ & $-3,461$ \\
\hline
\end{tabular}

Fuente: Elaboración propia.

Cuadro A2.2

Resultados del análisis de vectores autorregresivos: factor número 1, índice de noticias conflictivas estandarizado e índice de precios de los metales

\begin{tabular}{|c|c|c|c|c|c|c|}
\hline \multicolumn{7}{|c|}{ Vectores autorregresivos } \\
\hline \multicolumn{3}{|c|}{ Muestra: Trim 32003 a Trim 4 2015, pero con una interrupción } & \multicolumn{4}{|c|}{ Número de observaciones $=47$} \\
\hline \multicolumn{3}{|l|}{ Log likelihood = -216,7898 } & \multicolumn{4}{|c|}{$\mathrm{AlC}=9,480418$} \\
\hline \multicolumn{3}{|l|}{ Error de predicción final = 44,92575 } & \multicolumn{4}{|c|}{$\mathrm{HQIC}=9,569297$} \\
\hline \multicolumn{3}{|l|}{ Det (Sigma_ml) = 34,7905 } & \multicolumn{4}{|c|}{$\mathrm{SBIC}=9,716607$} \\
\hline Ecuación & Parms & RECM & R cuadrado & $x^{2}$ & $P>X^{2}$ & \\
\hline $\begin{array}{l}\text { D_índice_de_precios_de_los_metales } \\
\text { (primera diferencia) }\end{array}$ & 3 & 14,5725 & 0,0737 & 3,737807 & 0,1543 & \\
\hline \multirow[t]{2}{*}{ D_f1_scn (primera diferencia) } & 3 & 0,442755 & 0,2788 & 18,17191 & 0,0001 & \\
\hline & Coeficiente & Error estándar & z & $P>Z$ & [Intervalo de c & anza del 95\%] \\
\hline \multicolumn{7}{|l|}{$\begin{array}{l}\text { D_índice_de_precios_de_los_metales } \\
\text { (primera diferencia) }\end{array}$} \\
\hline \multicolumn{7}{|l|}{ índice_de_precios_de_los_metales } \\
\hline LD. (rezago primera diferencia) & 0,266852 & 0,145429 & 1,83 & 0,067 & $-0,01818$ & 0,551888 \\
\hline \multicolumn{7}{|l|}{ f1_sc } \\
\hline LD. (rezago primera diferencia) & $-4,57089$ & 4,260941 & $-1,07$ & 0,283 & $-12,9222$ & 3,780397 \\
\hline _cons & 2,260713 & 2,096179 & 1,08 & 0,281 & $-1,84772$ & 6,369148 \\
\hline \multicolumn{7}{|l|}{ D_f1_scn (primera diferencia) } \\
\hline \multicolumn{7}{|l|}{ índice_de_precios_de_los_metales } \\
\hline LD. (rezago primera diferencia) & 0,008484 & 0,004419 & 1,92 & 0,055 & $-0,00018$ & 0,017144 \\
\hline \multicolumn{7}{|l|}{ f1_sc } \\
\hline LD. (rezago primera diferencia) & $-0,54093$ & 0,12946 & $-4,18$ & 0,000 & $-0,79466$ & $-0,28719$ \\
\hline _cons & $-0,0027$ & 0,063688 & $-0,04$ & 0,966 & $-0,12752$ & 0,122131 \\
\hline
\end{tabular}

Fuente: Elaboración propia.

Nota: AIC: criterio de información de Akaike; HQIC: criterio de información de Hannan-Quinn; SBIC: criterio de información bayesiano de Schwarz; RECM: raíz del error cuadrático medio. 
Cuadro A2.3

Regresiones de efectos fijos por país y año del índice de noticias conflictivas violentas estandarizado

\begin{tabular}{|c|c|c|c|c|c|c|}
\hline Variables & $\begin{array}{c}1 \\
\text { In } \\
\text { (índice de noticias conflictivas } \\
\text { violentas estandarizado (sv)) }\end{array}$ & $\begin{array}{c}2 \\
\text { In } \\
\text { (índice_sv) }\end{array}$ & $\begin{array}{c}3 \\
\text { In } \\
\text { (índice_sv) }\end{array}$ & $\begin{array}{c}4 \\
\text { In } \\
\text { (índice_sv) }\end{array}$ & $\begin{array}{c}5 \\
\text { In } \\
\text { (índice_sv) }\end{array}$ & $\begin{array}{c}6 \\
\text { In } \\
\text { (índice_sv) }\end{array}$ \\
\hline \multirow[t]{2}{*}{$\begin{array}{l}\text { In(rentas procedentes de la minería como } \\
\text { porcentaje del PBI) }\end{array}$} & 0,0344 & 0,0393 & 0,0866 & & & \\
\hline & $(0,104)$ & $(0,107)$ & $(0,106)$ & & & \\
\hline \multirow[t]{2}{*}{ In(índice_de_precios_de_los_metales) } & & & & $-0,0108$ & 0,00591 & 0,0232 \\
\hline & & & & $(0,103)$ & $(0,129)$ & $(0,103)$ \\
\hline \multirow[t]{2}{*}{$\begin{array}{l}\text { In(PBI per cápita en dólares a valores } \\
\text { constantes de 2010) }\end{array}$} & & $-0,130$ & & & $-0,101$ & \\
\hline & & $(0,470)$ & & & $(0,458)$ & \\
\hline \multirow[t]{2}{*}{$\begin{array}{l}\text { Primera diferencia_In(PBI per cápita en } \\
\text { dólares a valores constantes de 2010) }\end{array}$} & & & $-4,425^{\star}$ & & & $-3,809$ \\
\hline & & & $(2,612)$ & & & $(2,488)$ \\
\hline \multirow[t]{2}{*}{ Constante } & $-1,215^{\star \star \star}$ & 0,0467 & $-1,120^{\star \star \star}$ & $-1,173^{\star *}$ & $-0,260$ & $-1,235^{\star *}$ \\
\hline & $(0,0985)$ & $(4,572)$ & $(0,111)$ & $(0,496)$ & $(4,169)$ & $(0,489)$ \\
\hline Observaciones & 52 & 52 & 52 & 52 & 52 & 52 \\
\hline R cuadrado & 0,382 & 0,383 & 0,430 & 0,380 & 0,381 & 0,419 \\
\hline Efectos por país y año & Sí & Sí & Sí & Sí & Sí & Sí \\
\hline Número de países & 4 & 4 & 4 & 4 & 4 & 4 \\
\hline
\end{tabular}

Fuente: Elaboración propia.

Nota: Significación estadística del coeficiente: ${ }^{\star \star \star} p<0,01,{ }^{\star \star} p<0,05,{ }^{\star} p<0,1$. Los errores estándares robustos se muestran entre paréntesis. 